\title{
Second-Order Optimal Array Receivers for Synchronization of BPSK, MSK, and GMSK Signals Corrupted by Noncircular Interferences
}

\author{
Pascal Chevalier, François Pipon, and François Delaveau \\ Thales-Communications, EDS/SPM, 160 Bd Valmy, 92704 Colombes Cedex, France
}

Received 4 October 2006; Revised 16 March 2007; Accepted 13 May 2007

Recommended by Benoit Champagne

\begin{abstract}
The synchronization and/or time acquisition problem in the presence of interferences has been strongly studied these last two decades, mainly to mitigate the multiple access interferences from other users in DS/CDMA systems. Among the available receivers, only some scarce receivers may also be used in other contexts such as F/TDMA systems. However, these receivers assume implicitly or explicitly circular (or proper) interferences and become suboptimal for noncircular (or improper) interferences. Such interferences are characteristic in particular of radio communication networks using either rectilinear (or monodimensional) modulations such as BPSK modulation or modulation becoming quasirectilinear after a preprocessing such as MSK, GMSK, or OQAM modulations. For this reason, the purpose of this paper is to introduce and to analyze the performance of second-order optimal array receivers for synchronization and/or time acquisition of BPSK, MSK, and GMSK signals corrupted by noncircular interferences. For given performances and in the presence of rectilinear signal and interferences, the proposed receiver allows a reduction of the number of sensors by a factor at least equal to two.
\end{abstract}

Copyright (c) 2007 Pascal Chevalier et al. This is an open access article distributed under the Creative Commons Attribution License, which permits unrestricted use, distribution, and reproduction in any medium, provided the original work is properly cited.

\section{INTRODUCTION}

The synchronization and/or time acquisition problem in the presence of interferences has been strongly studied these last two decades, mainly to mitigate the multiple access interferences (MAI) from other users in DS/CDMA systems. The available receivers may be implemented from either monoantenna [1-7] or multi-antennas [8-12]. Receivers presented in $[9,12]$ are analog receivers while the other ones are digital receivers. Most of the available digital receivers are very specific of the CDMA context and cannot be used elsewhere, since they require assumptions such as a spreading sequence which is repeated at each symbol [1-7], a very large number of MAI [11], no data on the codes $[8,11]$ or periodic and orthogonal sequences [8]. On the other hand, [5], which does not require the previous assumptions, assumes interferences with known delays and spreading sequences, which corresponds to very specific situations. On the contrary, although assuming orthogonal and periodic codes, maximum likelihood (ML) receivers presented in [10] belong to the family of the scarce receivers which may be used in other contexts than DS/CDMA systems such as F/TDMA systems in par- ticular. These receivers also consider random data modulating the code and generalize the least square (LS) approach presented in [8]. However, receivers presented in [10] assume stationary, and then second-order (SO) circular [13] (or proper [14]) Gaussian interferences. Moreover, they do not use any of the structure in the latter, although this structure is perfectly known for interferences generated by the system itself. In particular, receivers presented in $[8,10]$ become sub-optimal for SO noncircular (or improper [15]) interferences. This property is characteristic of radio communication networks using either rectilinear (or monodimensional) modulations, such as amplitude modulation (AM), amplitude phase shift keying (ASK), binary phase shift keying (BPSK) modulations, or modulations becoming quasirectilinear after a preprocessing such as Minimum Shift Keying (MSK), Gaussian MSK (GMSK), or offset quadrature amplitude modulations (OQAM) [16]. The BPSK modulation is still of interest for various current wireless systems [15], whereas MSK and GMSK modulations may be interpreted as a BPSK modulation after a simple algebraic operation of derotation on the baseband signal [17-19]. For these reasons, the first purpose of this paper is to introduce and to 
analyze the performance of the SO optimal array receiver for synchronization and/or time acquisition of BPSK signals corrupted by noncircular, and more precisely by rectilinear interferences. This receiver, patented recently [20], implements an optimal, in an LS sense, widely linear (WL) [21] spatial filtering of the data followed by a correlation operation with a training sequence. Extensions of these results to MSK and GMSK signals [16] are presented at the end of the paper and constitute the second purpose of this paper.

The first use of WL filters in signal processing has been reported in [22], the first discussion about their interest for cyclostationary signals has been introduced in $[23,24]$ and the proof of their optimality in SO noncircular context has been presented in $[21,25,26]$. Since the previous papers, optimal WL filtering has raised an increasing interest this last decade in radio communications for demodulation purposes (see [17] and references therein). However, up to now and to our knowledge, despite some works about frequency-offset estimation in noncircular contexts [27-29], optimal WL filtering has never been investigated for synchronization and/or time acquisition purposes in noncircular contexts, hence the present paper. Note that some results of the paper have already been partially presented in the conference paper [30].

After an introduction of some notations, hypotheses, and data statistics in Section 2, the SO optimal array receiver for synchronization and/or time acquisition of a BPSK signal corrupted by noncircular interferences is presented in Section 3, where some general interpretations, properties, and performance of this receiver are described. Some insigths into the performance of the latter in the presence of one rectilinear interference are presented and illustrated in Section 4. Section 5 investigates extensions of the previous results to MSK and GMSK signals. Finally Section 6 concludes the paper.

\section{HYPOTHESES AND PROBLEM FORMULATION FOR BPSK SIGNALS}

\subsection{Hypotheses}

We consider an array of $N$ narrowband (NB) sensors receiving the contribution of a BPSK signal and a total noise composed of some potentially SO noncircular interferences and a background noise. This situation is, for example, characteristic of a BPSK radio communication network where interferences correspond to cochannel interferences (CCI) generated by the network itself. The complex envelope of the useful BPSK signal is, to within a constant, given by

$$
s(t)=\sum_{n} a_{n} v(t-n T),
$$

where $a_{n}= \pm 1$ is the transmitted symbol $n, T$ is the symbol duration, and $v(t)$ is a real-valued pulse-shaped filter such that $r_{v}(t) \triangleq v(t) \otimes v(-t)^{*}$ is a Nyquist filter, that is, $r_{v}(n T)=0$ for $n \neq 0$. Symbols $\otimes$ and $*$ are the convolution and the complex conjugation operations, respectively. Note that $r_{v}(t)$ is the autocorrelation of $v(t)$ and the previous condition is verified if $v(t)$ is either a raised cosine pulse-shaped filter or a rectangular pulse of duration $T$. In most of radio communication systems, $K$ training symbols $a_{n}(0 \leq n \leq K-1)$ are periodically transmitted among information symbols for synchronization and/or time acquisition purposes. These $K$ training symbols are known by the receiver and can be considered as deterministic symbols. On the contrary, the information symbols are unknown by the receiver, are random and can be considered as i.i.d stationary symbols. For example, in a burst transmission, one training sequence of $K$ symbols jointly with some information symbols are transmitted at each burst. Assuming a useful propagation channel with $M$ multipaths, noting $\mathbf{x}(t)$ the vector of the complex envelopes of the signals at the output of the sensors, $T_{e}$ the sample period such that $T / T_{e}$ is an integer $q, s_{v}\left(k T_{e}\right) \triangleq s(t) \otimes v(-t)^{*} / t=k T_{e}$ and $\mathbf{x}_{v}\left(k T_{e}\right) \triangleq$ $\mathbf{x}(t) \otimes v(-t)^{*} / t=k T_{e}$ the sampled useful signal and observation vector at the output of the matched filter $v(-t)^{*}$, we obtain

$$
\mathbf{x}_{v}\left(k T_{e}\right) \approx \sum_{i=0}^{M-1} \mu_{s} s_{v}\left(k T_{e}-\tau_{i}\right) \mathbf{h}_{s i}+\mathbf{b}_{T v}\left(k T_{e}\right)
$$

In this equation, $\mu_{s}$ is a real parameter controlling the transmitted amplitude of the useful signal, $\tau_{i}$ and $\mathbf{h}_{s i}$ are the delay and the channel vector of the useful path $i, \mathbf{b}_{T v}\left(k T_{e}\right)$ is the sampled total noise vector at the output of $v(-t)^{*}$, which contains the contribution of interferences and background noise and which is assumed to be uncorrelated with all the signals $s_{v}\left(k T_{e}-\tau_{i}\right)$. In a digital radio communication system, the synchronization function aims at detecting the different useful paths (interception) and estimating their delays $\tau_{i}$ (time acquisition). For equalization/demodulation purposes, it aims also at choosing the best sampling time, from the estimated power of each detected path, and at optimally positioning the equalizer with respect to the delays of the detected paths. The synchronization process is thus a joint detection and estimation problem. Of course, the probability to improve the best sampling time increases with the degree of data oversampling. In such a context, there is no need to exactly estimate the delays $\tau_{i}(0 \leq i \leq M-1)$ and the problem rather consists, for each useful path $i_{0}$, to detect the most powerful sample associated with this path. More precisely, for each useful path $i_{0}$, noting $l_{o} T_{e}$ the sample time which is the nearest of $\tau_{i 0}$, the problem considered in this paper is both to detect the presence of the useful path $i_{0}$ and to find the best estimate of $l_{o} T_{e}$ from the sampled observation vectors. Assuming an optimal sampling time for the path $i_{0}$, the sampled observation vector considered in practice can then be written as

$$
\mathbf{x}_{v}\left(k T_{e}\right) \approx \mu_{s} s_{v}\left(\left(k-l_{o}\right) T_{e}\right) \mathbf{h}_{s}+\mathbf{b}_{T v}\left(k T_{e}\right)^{\prime} .
$$

In this equation, $\mathbf{h}_{s}$ is the channel vector of the useful path $i_{0}$ and $\mathbf{b}_{T v}\left(k T_{e}\right)^{\prime}$ is the sampled contribution of both the total noise vector $\mathbf{b}_{T v}\left(k T_{e}\right)$ and the useful paths different from $i_{0}$. Note that $\mathbf{b}_{T v}\left(k T_{e}\right)^{\prime}=\mathbf{b}_{T v}\left(k T_{e}\right)$ for a useful propagation channel with no delay spread, which occurs, for example, for free space propagation (reception from satellite, plane or unmanned aerial vehicle) or flat fading channels (some reception situations for urban radio communications). Besides, to simplify the developments of the paper, model (3) 
assumes that the carrier frequency of the useful signal is a priori known (which is true for cellular networks) or has been perfectly compensated.

\subsection{Second-order statistics of the data}

The SO statistics of the data considered in the following correspond to the first and second correlation matrix of $\mathbf{x}_{v}\left(k T_{e}\right)$, defined by $R_{x}\left(k T_{e}\right) \triangleq \mathrm{E}\left[\mathbf{x}_{v}\left(k T_{e}\right) \mathbf{x}_{v}\left(k T_{e}\right)^{\dagger}\right]$ and $C_{x}\left(k T_{e}\right) \triangleq \mathrm{E}\left[\mathbf{x}_{v}\left(k T_{e}\right) \quad \mathbf{x}_{v}\left(k T_{e}\right)^{\mathrm{T}}\right]$, respectively, where $\mathrm{T}$ and $\dagger$ correspond to the transposition and transposition conjugation operation respectively. In a same way, the first and second correlation matrix of $\mathbf{b}_{T v}\left(k T_{e}\right)$ are defined by $R\left(k T_{e}\right) \triangleq \mathrm{E}\left[\mathbf{b}_{T v}\left(k T_{e}\right) \mathbf{b}_{T v}\left(k T_{e}\right)^{\dagger}\right]$ and $C\left(k T_{e}\right) \triangleq \mathrm{E}\left[\mathbf{b}_{T v}\left(k T_{e}\right) \mathbf{b}_{T v}\left(k T_{e}\right)^{\mathrm{T}}\right]$, respectively. The first and second correlation matrix of $\mathbf{b}_{T v}\left(k T_{e}\right)^{\prime}$ are defined by $R\left(k T_{e}\right)^{\prime} \triangleq \mathrm{E}\left[\mathbf{b}_{T v}\left(k T_{e}\right)^{\prime} \mathbf{b}_{T v}\left(k T_{e}\right)^{\prime \dagger}\right]$ and $C\left(k T_{e}\right)^{\prime} \triangleq$ $\mathrm{E}\left[\mathbf{b}_{T v}\left(k T_{e}\right)^{\prime} \mathbf{b}_{T v}\left(k T_{e}\right)^{\prime \mathrm{T}}\right]$ respectively. Note that $R\left(k T_{e}\right)^{\prime}=$ $R\left(k T_{e}\right)$ and $C\left(k T_{e}\right)^{\prime}=C\left(k T_{e}\right)$ for a useful propagation channel with no delay spread. Note also that $C\left(k T_{e}\right)=O$ (resp., $\left.C\left(k T_{e}\right)^{\prime}=O\right)$ for all $k$ for an SO circular vector $\mathbf{b}_{T v}\left(k T_{e}\right)$ (resp., $\left.\mathbf{b}_{T v}\left(k T_{e}\right)^{\prime}\right)$, where $O$ is the $(N \times N)$ zero matrix. Finally we note $\pi_{s}\left(k T_{e}\right) \triangleq \mathrm{E}\left[\left|s_{v}\left(k T_{e}\right)\right|^{2}\right]$ the instantaneous power of the transmitted useful signal for $\mu_{s}=1$. Note that the previous statistics depend on the time parameter since the considered useful signal and interferences are cyclostationary, due to their digital nature.

\subsection{Problem formulation}

Since the $K$ training symbols $a_{n}(0 \leq n \leq K-1)$, which are periodically transmitted for synchronization purposes, are known by the receiver, the associated useful samples $s_{v}(n T)=r_{v}(0) a_{n}(0 \leq n \leq K-1)$ are also known by the receiver. Then, a first way to solve the synchronization problem consists to find, for each useful path $i_{0}$, the best estimate, $\hat{l}_{o}$, of $l_{o}$. This can be done by searching for the integers $l$ for which the known useful samples $s_{v}(n T)(0 \leq n \leq K-1)$ are optimally estimated, in an LS sense, from the observation vectors $\mathbf{x}_{v}((l / q+n) T), 0 \leq n \leq K-1$. We solve this problem in Section 3.1, without any assumptions about the delay spread of the propagation channels, the orthogonality or the periodicity of the training sequence, contrary to $[8,10]$. A second way to solve the synchronization problem consists to optimally detect each useful path $i_{0}$. This can be done by searching for the integers $l$ for which the known useful samples $s_{v}(n T)(0 \leq n \leq K-1)$ are optimally detected from the observation vectors $\mathbf{x}_{v}((l / q+n) T), 0 \leq n \leq K-1$. We solve this problem in Section 3.2 under particular theoretical assumptions, showing off the hypotheses under which the two ways to solve the synchronization problem are equivalent to each other.

\section{OPTIMAL SYNCHRONIZATION FOR BPSK SIGNALS}

It is now well known $[17,21,25,26]$ that the linear filters are SO optimal for SO circular observations only but become sub-optimal in noncircular contexts for which the SO optimal filters are WL, weighting linearly and independently the observations and their complex conjugate. In these conditions, the first way to solve, in the presence of noncircular interferences, the synchronization problem presented in Section 2.3 is, for each useful path $i_{0}$, to search for the optimal integer $l$, noted $\hat{l}_{o}$, for which the known useful samples, $s_{v}(n T)=r_{v}(0) a_{n}(0 \leq n \leq K-1)$, are optimally estimated, in an LS sense, from a WL spatial filtering of the observation vectors $\mathbf{x}_{v}((l / q+n) T)(0 \leq n \leq K-1)$. This gives rise in Section 3.1 to the optimal LS array receiver, called OPT-LS receiver, for synchronization of the BPSK useful signal in the presence of noncircular interferences. This OPT-LS receiver is shown in Section 3.2 to also correspond, under some theoretical assumptions not required in practice, to the array receiver for which $\hat{l}_{o}$ allows the optimal detection, in terms of the generalized likelihood ratio test (GLRT) [31], of the known useful samples, $s_{v}(n T)(0 \leq n \leq K-1)$, from the observation vectors $\mathbf{x}_{v}\left(\left(\hat{l}_{o} / q+n\right) T\right)(0 \leq n \leq K-1)$. An enlightening interpretation and some performance of the OPTLS receiver are then presented in Sections 3.3 and 3.4, respectively. Note that the results presented in this section are completely new.

\subsection{Presentation of the OPT-LS receiver}

Synchronization or time acquisition from OPT-LS receiver consists to find, for each useful path $i_{0}$, the integer $l$, noted $\hat{l}_{o}$, which minimizes the LS error, $\hat{\mathcal{E}}_{\mathrm{WL}}\left(l T_{e}, K\right)$, between the known samples $s_{v}(n T)=r_{v}(0) a_{n}(0 \leq n \leq K-1)$ and their LS estimation from a WL spatial filtering of the data $\mathbf{x}_{v}((l / q+$ $n) T)(0 \leq n \leq K-1)$. The LS error, $\hat{\varepsilon}_{\mathrm{WL}}\left(l T_{e}, K\right)$, is defined by

$$
\widehat{\mathcal{E}}_{\mathrm{WL}}\left(l T_{e}, K\right) \triangleq \frac{1}{K} \sum_{n=0}^{K-1}\left|s_{v}(n T)-\widehat{\widetilde{\mathbf{w}}}\left(l T_{e}\right)^{\dagger} \widetilde{\mathbf{x}}_{v}\left(\left(\frac{l}{q}+n\right) T\right)\right|^{2}
$$

where $\tilde{\mathbf{x}}_{v}((l / q+n) T) \triangleq\left[\mathbf{x}_{v}((l / q+n) T)^{\mathrm{T}}, \mathbf{x}_{v}((l / q+n) T)^{\dagger}\right]^{\mathrm{T}}$ and where $\hat{\widetilde{\mathbf{w}}}\left(l T_{e}\right) \triangleq\left[\widehat{\mathbf{w}}_{1}\left(l T_{e}\right)^{\mathrm{T}}, \widehat{\mathbf{w}}_{2}\left(l T_{e}\right)^{\mathrm{T}}\right]^{\mathrm{T}}$ is the $(2 N \times 1)$ WL spatial filter which minimizes the criterion (4). This filter is defined by

$$
\hat{\tilde{\mathbf{w}}}\left(l T_{e}\right)=\left[\widehat{\mathbf{w}}_{1}\left(l T_{e}\right)^{\mathrm{T}}, \widehat{\mathbf{w}}_{1}\left(l T_{e}\right)^{\dagger}\right]^{\mathrm{T}}=\widehat{R}_{\widetilde{x}}\left(l T_{e}\right)^{-1} \hat{\mathbf{r}}_{\tilde{x} s}\left(l T_{e}\right),
$$

where the vector $\hat{\mathbf{r}}_{\tilde{x} s}\left(l T_{e}\right)$ and the matrix $\widehat{R}_{\widetilde{x}}\left(l T_{e}\right)$ are given by

$$
\begin{gathered}
\hat{\mathbf{r}}_{\tilde{x} s}\left(l T_{e}\right) \triangleq \frac{1}{K} \sum_{n=0}^{K-1} \tilde{\mathbf{x}}_{v}\left(\left(\frac{l}{q}+n\right) T\right) s_{v}(n T)^{*}, \\
\hat{R}_{\widetilde{x}}\left(l T_{e}\right) \triangleq \frac{1}{K} \sum_{n=0}^{K-1} \widetilde{\mathbf{x}}_{v}\left(\left(\frac{l}{q}+n\right) T\right) \tilde{\mathbf{x}}_{v}\left(\left(\frac{l}{q}+n\right) T\right)^{\dagger} .
\end{gathered}
$$

Using (5) to (7) into (4), we obtain a new expression of $\widehat{\varepsilon}_{\mathrm{WL}}\left(l T_{e}, K\right)$ given by

$$
\begin{aligned}
\hat{\mathcal{E}}_{\mathrm{WL}}\left(l T_{e}, K\right) & =\left[\frac{1}{K} \sum_{n=0}^{K-1}\left|s_{v}(n T)\right|^{2}\right]\left[1-\widehat{C}_{\mathrm{OPT}-\mathrm{LS}}\left(l T_{e}, K\right)\right] \\
& =\pi_{s}\left[1-\hat{C}_{\mathrm{OPT}-\mathrm{LS}}\left(l T_{e}, K\right)\right],
\end{aligned}
$$


where $\pi_{s} \triangleq r(0)^{2}$ is the input power of the useful BPSK samples, $s_{v}(n T)$, and $\hat{C}_{\mathrm{OPT}-\mathrm{LS}}\left(l T_{e}, K\right)$ such that $0 \leq \hat{\mathrm{C}}_{\mathrm{OPT} \text {-LS }} \times$ $\left(l T_{e}, K\right) \leq 1$ is given by

$$
\hat{C}_{\mathrm{OPT}-\mathrm{LS}}\left(l T_{e}, K\right) \triangleq\left(\frac{1}{\pi_{s}}\right) \hat{\mathbf{r}}_{\widetilde{x}}\left(l T_{e}\right)^{\dagger} \hat{R}_{\widetilde{x}}\left(l T_{e}\right)^{-1} \widehat{\mathbf{r}}_{\tilde{x}_{s}}\left(l T_{e}\right) .
$$

We deduce from (8) that for each useful path $i_{0}$, the parameter $\hat{l}_{o}$ locally maximizes the sufficient statistic $\hat{C}_{\mathrm{OPT}-\mathrm{LS}}\left(l T_{e}, K\right)$ given by (9). As a consequence, the estimated sampled delays of all the useful paths correspond to the sample times $l T_{e}$ for which $\hat{C}_{\mathrm{OPT}-\mathrm{LS}}\left(l T_{e}, K\right)$ is locally maximum. If the number, $M$, of useful paths is a priori known, their estimated sampled delays correspond to the positions of the $M$ maxima of $\hat{C}_{\text {OPT-LS }}\left(l T_{e}, K\right)$. However, if $M$ is not known a priori, a threshold has to be introduced to limit the false alarm rate (FAR). In these conditions, the estimated sampled delays of the useful paths correspond to the sample times $l T_{e}$ for which $\hat{C}_{\text {OPT-LS }}\left(l T_{e}, K\right)$ is locally maximum and above the threshold. The approach considered in this Section 3.1 does not require any assumption about the propagation channels, the interferences and the training sequence. Thus, in practice, OPT-LS receiver may be used for synchronization or time acquisition in the presence of arbitrary propagation channels and interferences. Note that the receiver presented in [8] for the same problem, called conventional LS array receiver and noted CONV-LS receiver in the following, is deduced from a similar LS approach but takes into account only a linear spatial filtering of the data, $\mathbf{x}_{v}((l / q+n) T)(0 \leq n \leq K-1)$, instead of a WL one. It gives rise to the conventional sufficient statistic $\hat{C}_{\mathrm{CONV}-\mathrm{LS}}\left(l T_{e}, K\right)$ such that $0 \leq \hat{C}_{\mathrm{CONV}-\mathrm{LS}}\left(l T_{e}, K\right) \leq 1$, defined by

$$
\hat{C}_{\mathrm{CONV}-\mathrm{LS}}\left(l T_{e}, K\right) \triangleq\left(\frac{1}{\pi_{s}}\right) \hat{\mathbf{r}}_{x s}\left(l T_{e}\right)^{\dagger} \hat{R}_{x}\left(l T_{e}\right)^{-1} \hat{\mathbf{r}}_{x s}\left(l T_{e}\right),
$$

where the vector $\hat{\mathbf{r}}_{x s}\left(l T_{e}\right)$ and the matrix $\hat{R}_{x}\left(l T_{e}\right)$ are defined by (6) and (7), respectively but where the vector $\tilde{\mathbf{x}}_{v}((l / q+$ $n) T)$ is replaced by $\mathbf{x}_{v}((l / q+n) T)$. This conventional receiver is the heart of the interference analyzer described in [32] for the GSM network monitoring.

\subsection{Interpretation of OPT-LS and CONV-LS receivers in terms of GLRT-based detectors}

\subsubsection{Theoretical assumptions}

In this section, we present the assumptions under which OPT-LS and CONV-LS receivers for $l=l_{o}$ also correspond to the GLRT-based receiver for the detection of the known samples $s_{v}(n T)=r_{v}(0) a_{n}(0 \leq n \leq K-1)$ from the observation vectors $\mathbf{x}_{v}\left(\left(l_{0} / q+n\right) T\right)(0 \leq n \leq K-1)$. Note that these assumptions are theoretical, are not necessarily verified in practical situations and are absolutely not required in practice to successfully implement the conventional and optimal receivers defined by (10) and (9), respectively. However, these assumptions allow in particular to get more insights into the situations for which (9) and (10) become optimal from a GLRT-based detection point of view. Besides, they allow to show off the optimality of (9) and (10) in the presence of SO noncircular and circular total noise, respectively. Defining the vector $\tilde{\mathbf{b}}_{T v}((l / q+n) T)$ by $\tilde{\mathbf{b}}_{T v}((l / q+n) T) \triangleq\left[\mathbf{b}_{T v}((l / q+n) T)^{\mathrm{T}}, \mathbf{b}_{T v}((l / q+n) T)^{\dagger}\right]^{\mathrm{T}}$, these theoretical assumptions correspond to the following.

(A1) The samples $\tilde{\mathbf{b}}_{T v}\left(\left(l_{o} / q+n\right) T\right), 0 \leq n \leq K-1$ are uncorrelated to each other.

(A2) The matrices $R\left(\left(l_{0} / q+n\right) T\right)$ and $C\left(\left(l_{o} / q+n\right) T\right)$ do not depend on the symbol indice $n$.

(A3) The matrices $R\left(\left(l_{o} / q+n\right) T\right), C\left(\left(l_{o} / q+n\right) T\right)$ and the vector $\mathbf{h}_{s}$ are unknown.

(A4) The samples $\mathbf{b}_{T v}\left(\left(l_{0} / q+n\right) T\right), 0 \leq n \leq K-1$, are Gaussian.

(A5) The samples $\mathbf{b}_{T v}\left(\left(l_{o} / q+n\right) T\right), 0 \leq n \leq K-1$, are SO noncircular.

(A6) The samples $\mathbf{b}_{T v}\left(\left(l_{o} / q+n\right) T\right)$ and $s_{v}(m T), 0 \leq n, m \leq$ $K-1$, are statistically independent.

(A7) The useful propagation channel has no delay spread $\left(\mathbf{b}_{T v}\left(\left(l_{0} / q+n\right) T\right)^{\prime}=\mathbf{b}_{T v}\left(\left(l_{o} / q+n\right) T\right)\right)$.

Note that contrary to $[8,10]$, no assumption is made about the correlation properties of the training sequence. (A1) would only be true for interference propagation channels with no delay spread as soon as the rectilinear interferences would be generated by the network itself (internal BPSK interferences) and would be synchronous with the useful signal to verify the Nyquist criterion. (A2) would be true for cyclostationary interferences with symbol period $T$, as it would be the case for internal BPSK interferences. (A4) could not be verified in the presence of rectilinear interferences and would be a false assumption allowing to only exploit the SO statistics of the observations from a GLRT approach. (A5) would be true in the presence of rectilinear interferences in particular but is generally not exploited in detection problems. (A6) would always be verified due to the deterministic character of $s_{v}(m T)(0 \leq m \leq K-1)$ jointly with the zero-mean and random character of the total noise. Finally, (A7) would be valid for some particular applications.

\subsubsection{GLRT-based receiver for detection}

To compute the GLRT-based receiver for detection, we consider the optimal delay $l_{o} T_{e}$ and the detection problem with two hypotheses $\mathrm{H} 0$ and $\mathrm{H} 1$, where $\mathrm{H} 0$ and $\mathrm{H} 1$ correspond to the presence of total noise only and signal plus total noise into the observation vector $\mathbf{x}_{v}\left(\left(l_{0} / q+n\right) T\right)$, respectively. Under these two hypotheses, using (2), (3), and (A7), the vector $\mathbf{x}_{v}\left(\left(l_{0} / q+n\right) T\right)$ can be written as

$$
\begin{aligned}
& \mathrm{H} 1: \mathbf{x}_{v}\left(\left(\frac{l_{o}}{q}+n\right) T\right) \approx \mu_{s} s_{v}(n T) \mathbf{h}_{s}+\mathbf{b}_{T v}\left(\left(\frac{l_{o}}{q}+n\right) T\right), \\
& \mathrm{H} 0: \mathbf{x}_{v}\left(\left(\frac{l_{o}}{q}+n\right) T\right) \approx \mathbf{b}_{T v}\left(\left(\frac{l_{o}}{q}+n\right) T\right) .
\end{aligned}
$$

According to the Neyman-Pearson theory of detection [31] and using (A6), the optimal receiver for detection of samples $s_{v}(n T)$ from $\mathbf{x}_{v}\left(\left(l_{0} / q+n\right) T\right)$ over the training sequence duration is the likelihood ratio (LR) receiver, which consists 
to compare to a threshold the function $\operatorname{LR}\left(l_{o} T_{e}, K\right)$ defined by

$$
\mathrm{LR}\left(l_{o} T_{e}, K\right) \triangleq \frac{\mathrm{p}\left[\mathbf{x}_{v}\left(\left(l_{o} / q+n\right) T\right), 0 \leq n \leq K-1, / \mathrm{H} 1\right]}{\mathrm{p}\left[\mathbf{x}_{v}\left(\left(l_{o} / q+n\right) T\right), 0 \leq n \leq K-1, / \mathrm{H} 0\right]}
$$

In (12), $\mathrm{p}\left[\mathbf{x}_{v}\left(\left(l_{o} / q+n\right) T\right), 0 \leq n \leq K-1, / \mathrm{Hi}\right](i=0,1)$ is the conditional probability density of $\left[\mathbf{x}_{v}\left(l_{o} T_{e}\right), \mathbf{x}_{v}\left(l_{o} T_{e}+\right.\right.$ $\left.T), \ldots, \mathbf{x}_{v}\left(l_{o} T_{e}+(K-1) T\right)\right]^{\mathrm{T}}$ under Hi. Using (11) into (12), and recalling that $s_{v}(n T)$ is a deterministic quantity, we get

$$
\mathrm{LR}\left(l_{o} T_{e}, K\right) \triangleq \frac{\mathrm{p}\left[A^{\prime}\right]}{\mathrm{p}\left[B^{\prime}\right]}
$$

(where $A^{\prime}=\left\{\mathbf{b}_{T v}\left(\left(l_{o} / q+n\right) T\right)=\mathbf{x}_{v}\left(\left(l_{o} / q+n\right) T\right)-\mu_{s} s_{v}(n T) \mathbf{h}_{s}\right.$, $0 \leq n \leq K-1\}$, and $B^{\prime}=\left\{\mathbf{b}_{T v}\left(\left(l_{o} / q+n\right) T\right)=\mathbf{x}_{v}\left(\left(l_{o} / q+n\right) T\right)\right.$, $0 \leq n \leq K-1\})$.

Using (A1), (A2), and (A4), expression (13) takes the form

$$
\operatorname{LR}\left(l_{o} T_{e}, K\right)=\frac{\prod_{n=0}^{K-1} \mathrm{p}\left[S_{n}^{\prime}\right]}{\prod_{n=0}^{K-1} \mathrm{p}\left[D_{n}^{\prime}\right]}
$$

$\left(S_{n}^{\prime}=\left\{\mathbf{b}_{T v}\left(\left(l_{o} / q+n\right) T\right)=\mathbf{x}_{v}\left(\left(l_{o} / q+n\right) T\right)-\mu_{s} s_{v}(n T) \mathbf{h}_{s} / s_{v}(n T)\right.\right.$, $\left.\mu_{s} \mathbf{h}_{s}, R\left(l_{o} T_{e}\right), C\left(l_{o} T_{e}\right)\right\}, D_{n}^{\prime}=\left\{\mathbf{b}_{T v}\left(\left(l_{o} / q+n\right) T\right)=\mathbf{x}_{v}\left(\left(l_{o} / q+\right.\right.\right.$ n) $\left.\left.T) / R\left(l_{o} T_{e}\right), C\left(l_{o} T_{e}\right)\right\}\right)$.

From (A2), (A4), and (A5), the probability density of $\mathbf{b}_{T v}\left(\left(l_{o} / q+n\right) T\right)$ becomes a function of $\tilde{\mathbf{b}}_{T v}\left(\left(l_{o} / q+n\right) T\right)$ given by $[33,34]$

$$
\begin{aligned}
& \mathrm{p}\left[\tilde{\mathbf{b}}_{T v}\left(\left(\frac{l_{o}}{q}+n\right) T\right)\right] \triangleq \pi^{-N} \operatorname{det}\left[R_{\tilde{b}}\left(l_{o} T_{e}\right)\right]^{-1 / 2} \\
& \times \exp \left[-\left(\frac{1}{2}\right) \tilde{\mathbf{b}}_{T v}\left(\left(\frac{l_{o}}{q}+n\right) T\right)^{\dagger}\right. \\
&\left.\times R_{\widetilde{b}}\left(l_{o} T_{e}\right)^{-1} \widetilde{\mathbf{b}}_{T v}\left(\left(\frac{l_{o}}{q}+n\right) T\right)\right] .
\end{aligned}
$$

Using (15) into (14), we obtain

$$
\operatorname{LR}\left(l_{o} T_{e}, K\right)=\frac{\prod_{n=0}^{K-1} \mathrm{p}\left[E_{n}^{\prime}\right]}{\prod_{n=0}^{K-1} \mathrm{p}\left[F_{n}^{\prime}\right]}
$$

$\left(E_{n}^{\prime}=\left\{\tilde{\mathbf{b}}_{T v}\left(\left(l_{o} / q+n\right) T\right)=\tilde{\mathbf{x}}_{v}\left(\left(l_{o} / q+n\right) T\right)-\mu_{s} s_{v}(n T) \tilde{\mathbf{h}}_{s} / s_{v}(n T)\right.\right.$, $\left.\mu_{s} \tilde{\mathbf{h}}_{s}, R_{\tilde{b}}\left(l_{o} T_{e}\right)\right\}, F_{n}^{\prime}=\left\{\tilde{\mathbf{b}}_{T v}\left(\left(l_{o} / q+n\right) T\right)=\tilde{\mathbf{x}}_{v}\left(\left(l_{o} / q+n\right) T\right) /\right.$ $\left.\left.R_{\tilde{b}}\left(l_{o} T_{e}\right)\right\}\right)$, and $\tilde{\mathbf{h}}_{s} \triangleq\left[\mathbf{h}_{s}^{\mathrm{T}}, \mathbf{h}_{s}{ }^{\dagger}\right]^{\mathrm{T}}$ and where $R_{\tilde{b}}\left(l_{o} T_{e}\right)$ is defined by

$$
\begin{aligned}
R_{\tilde{b}}\left(l_{o} T_{e}\right) & \triangleq \mathrm{E}\left[\tilde{\mathbf{b}}_{T v}\left(\left(\frac{l_{o}}{q}+n\right) T\right)\right. \\
& =\left(\begin{array}{cc}
R\left(l_{o} T_{e}\right) & C\left(l_{o} T_{e}\right) \\
C\left(l_{o} T_{e}\right)^{*} & R\left(l_{o} T_{e}\right)^{*}
\end{array}\right) .
\end{aligned}
$$

Note that matrix $R_{\tilde{b}}\left(l_{o} T_{e}\right)$ contains the information about the potential noncircularity of the total noise through the matrix $C\left(l_{o} T_{e}\right)$, which is not zero for SO noncircular total noise. As, from (A3), $\mu_{s} \tilde{\mathbf{h}}_{s}$ and $R_{\widetilde{b}}\left(l_{o} T_{e}\right)$ are assumed to be unknown, they have to be replaced in (16) by their maximum likelihood (ML) estimates, giving rise to a GLRT approach. In these conditions, it is shown in the appendix that a sufficient statistic for the optimal detection, from a GLRT point of view, of $s_{v}(n T)(0 \leq n \leq K-1)$ from the observation vectors $\mathbf{x}_{v}\left(\left(l_{o} / q+n\right) T\right)(0 \leq n \leq K-1)$, is, under the assumptions (A1) to (A7), given by $\hat{C}_{\mathrm{OPT}-\mathrm{LS}}\left(l_{o} T_{e}, K\right)$ defined by (9). We deduce from the previous results that, under the theoretical assumptions (A1) to (A7), not necessarily verified and not required in practice, the optimal synchronization and time acquisition of the useful BPSK signal from the GLRT approach consists to compute, for each sample time $l T_{e}$, the quantity $\hat{C}_{\mathrm{OPT}-\mathrm{LS}}\left(l T_{e}, K\right)$, defined by (9), and to compare it to a threshold. The sampled delays of the useful paths thus correspond to the sample times $l T_{e}$ which generate local maximum values of $\hat{C}_{\mathrm{OPT}-\mathrm{LS}}\left(l T_{e}, K\right)$ among those which are over the threshold. Thus theoretical assumptions (A1) to (A7) allow to give conditions of optimality of the OPTLS receiver, in the GLRT sense, among which we find the condition of SO noncircularity of the total noise, valid for rectilinear interferences in particular. Nevertheless, when at least one of the assumptions (A1) to (A7) is not verified, as it may be the case for most practical situations, receiver (9) is no longer optimal in terms of detection but this does not mean that it does not work in practice. Note finally that a similar GLRT approach, but made under the theoretical assumptions (A1bis), (A2), (A3), (A4), (A5bis), (A6) and (A7), where (A1bis) and (A5bis) are defined by

(A1bis) the samples $\mathbf{b}_{T v}\left(\left(l_{o} / q+n\right) T\right), 0 \leq n \leq K-1$, are uncorrelated to each other,

(A5bis) the samples $\mathbf{b}_{T v}\left(\left(l_{o} / q+n\right) T\right), 0 \leq n \leq K-1$, are SO circular,

is reported in [10] and gives rise to the sufficient statistic $\widehat{C}_{\text {CONV-LS }}\left(l_{o} T_{e}, K\right)$ defined by (10). This shows that (10) is directly related to a (false) circular total noise assumption and becomes sub-optimal for noncircular total noise.

\subsection{Enlightening interpretation}

Using (5) into (9) and the fact that $s_{v}(n T)=s_{v}(n T)^{*}$ for BPSK useful signals, it is easy to verify that, whatever the propagation channel is, the statistic $\hat{C}_{\mathrm{OPT}-\mathrm{LS}}\left(l T_{e}, K\right)$ defined by (9), which is a real quantity, takes the form

$$
\widehat{C}_{\mathrm{OPT}-\mathrm{LS}}\left(l T_{e}, K\right)=\left(\frac{1}{K \pi_{s}}\right) \sum_{n=0}^{K-1} y_{v \mathrm{WL}}\left(\left(\frac{l}{q}+n\right) T\right) s_{v}(n T),
$$

where $y_{v \mathrm{WL}}((l / q+n) T) \triangleq \hat{\tilde{\mathbf{w}}}\left(l T_{e}\right)^{\dagger} \widetilde{\mathbf{x}}_{v}((l / q+n) T)=$ $2 \operatorname{Re}\left[\widehat{\mathbf{w}}_{1}\left(l T_{e}\right)^{\dagger} \mathbf{x}_{v}((l / q+n) T)\right]$ is also a real quantity. Expression (18) shows that the sufficient statistic $\hat{C}_{\mathrm{OPT}-\mathrm{LS}}\left(l T_{e}, K\right)$ corresponds, to within a normalization factor, to the result of the correlation between the training sequence, $s_{v}(n T)$, and 
the output, $y_{v \mathrm{WL}}((l / q+n) T)$, of the WL spatial filter $\hat{\tilde{\mathbf{w}}}\left(l T_{e}\right)$ (5) as it is illustrated in Figure 1.

The filter $\hat{\widetilde{\mathbf{w}}}\left(l T_{e}\right)$ is an estimate of the WL filter $\tilde{\mathbf{w}}\left(l T_{e}\right)$ which minimizes the time-averaged mean square error (MSE), $\varepsilon_{\mathrm{WL}}\left(l T_{e}, \tilde{\mathbf{w}}\right)$, over $K$ observation samples, between $s_{v}(n T)$ and the real output $\tilde{\mathbf{w}}^{\dagger} \widetilde{\mathbf{x}}_{v}((l / q+n) T)=2 \operatorname{Re} \times$ $\left[\mathbf{w}^{\dagger} \mathbf{x}_{v}((l / q+n) T)\right]$, defined by

$$
\varepsilon_{\mathrm{WL}}\left(l T_{e}, \tilde{\mathbf{w}}\right) \triangleq \frac{1}{K} \sum_{n=0}^{K-1} \mathrm{E}\left[\left|s_{v}(n T)-\tilde{\mathbf{w}}^{\dagger} \widetilde{\mathbf{x}}_{v}\left(\left(\frac{l}{q}+n\right) T\right)\right|^{2}\right]
$$

where $\tilde{\mathbf{w}} \triangleq\left[\mathbf{w}^{\mathrm{T}}, \mathbf{w}^{\dagger}\right]^{\mathrm{T}}$. The filter $\tilde{\mathbf{w}}\left(l T_{e}\right)$ is thus defined by $\tilde{\mathbf{w}}\left(l T_{e}\right) \triangleq R_{\tilde{x}, \mathrm{av}}\left(l T_{e}\right)^{-1} \mathbf{r}_{\tilde{x} s, \mathrm{av}}\left(l T_{e}\right)=\left[\mathbf{w}_{1}\left(l T_{e}\right)^{\mathrm{T}}, \mathbf{w}_{1}\left(l T_{e}\right)^{\dagger}\right]^{\mathrm{T}}$, where $\mathbf{r}_{\tilde{x} s, \text { av }}\left(l T_{e}\right)$ and $R_{\tilde{x} \text {,av }}\left(l T_{e}\right)$ are defined by

$$
\begin{gathered}
\mathbf{r}_{\tilde{x} s, \mathrm{av}}\left(l T_{e}\right) \triangleq \frac{1}{K} \sum_{n=0}^{K-1} \mathrm{E}\left[\tilde{\mathbf{x}}_{v}\left(\left(\frac{l}{q}+n\right) T\right) s_{v}(n T)^{*}\right] \\
R_{\tilde{x}, \mathrm{av}}\left(l T_{e}\right) \triangleq \frac{1}{K} \sum_{n=0}^{K-1} \mathrm{E}\left[\tilde{\mathbf{x}}_{v}\left(\left(\frac{l}{q}+n\right) T\right) \tilde{\mathbf{x}}_{v}\left(\left(\frac{l}{q}+n\right) T\right)^{\dagger}\right]
\end{gathered}
$$

As a consequence, $\hat{C}_{\mathrm{OPT} \text {-LS }}\left(l T_{e}, K\right)$ is, to within a normalization factor, an estimate of the expected value of the correlation between the training samples $s_{v}(n T)$ and the outputs of $\tilde{\mathbf{w}}\left(l T_{e}\right)$, defined by

$$
\begin{aligned}
C_{\mathrm{OPT}-\mathrm{LS}}\left(l T_{e}, K\right) \\
=\left(\frac{1}{K \pi_{s}}\right) \sum_{n=0}^{K-1} \mathrm{E}\left[\tilde{\mathbf{w}}\left(l T_{e}\right)^{\dagger} \tilde{\mathbf{x}}_{v}\left(\left(\frac{l}{q}+n\right) T\right) s_{v}(n T)\right] \\
=\frac{\mathbf{r}_{\tilde{x} s, \mathrm{av}}\left(l T_{e}\right)^{\dagger} R_{\tilde{x}, \mathrm{av}}\left(l T_{e}\right)^{-1} \mathbf{r}_{\tilde{x} s, \mathrm{av}}\left(l T_{e}\right)}{\pi_{s}} .
\end{aligned}
$$

Considering the detection or time acquisition of the useful path $i_{0}$, as long as $\tilde{\mathbf{b}}_{T v}((l / q+n) T)^{\prime}$ (in (3)) remains uncorrelated with $s_{v}(n T)$, which is in particular the case for a useful propagation channel with no delay spread, the vector $\mathbf{r}_{\tilde{x} s, \text { av }}\left(l T_{e}\right)$ can be written as

$$
\mathbf{r}_{\tilde{x} s, \mathrm{av}}\left(l T_{e}\right)=\frac{1}{K} \sum_{n=0}^{K-1} \mu_{s} \mathrm{E}\left[s_{v}\left(\left(\left(l-l_{o}\right) T_{e}\right)+n T\right) s_{v}(n T)^{*}\right] \tilde{\mathbf{h}}_{s} .
$$

This vector is collinear to $\widetilde{\mathbf{h}}_{s}$ and its norm is a function of $\left(l-l_{o}\right)$. In this context, as long as $l$ remains far from $l_{o}$, $\mathbf{r}_{\tilde{x} s, \text { av }}\left(l T_{e}\right)$, and thus $\tilde{\mathbf{w}}\left(l T_{e}\right)$, remain close to zero, which generates values of $C_{\mathrm{OPT}-\mathrm{LS}}\left(l T_{e}, K\right)$, and thus of $\hat{C}_{\mathrm{OPT} \text {-LS }}\left(l T_{e}, K\right)$, also close to zero to within the estimation noise due to the finite length of the training sequence for the latter. As $l$ gets close to $l_{o}$, the norm of $\mathbf{r}_{\tilde{x} s, \text { av }}\left(l T_{e}\right)$, and thus $C_{\mathrm{OPT}-\mathrm{LS}}\left(l T_{e}, K\right)$, increases and reaches its maximum value for $l=l_{o}$. In this case, the useful part of the observation vector $\widetilde{\mathbf{x}}_{v}\left(\left(l_{o} / q+n\right) T\right)$ and the training sequence $s_{v}(n T)$ are in phase and the filter $\tilde{\mathbf{w}}\left(l_{o} T_{e}\right)$ corresponds to the WL spatial matched filter (SMF) introduced in [17] and defined by

$$
\begin{aligned}
\tilde{\mathbf{w}}\left(l_{o} T_{e}\right) & =R_{\tilde{x}, \mathrm{av}}\left(l_{o} T_{e}\right)^{-1} \mathbf{r}_{\tilde{x} s, \mathrm{av}}\left(l_{o} T_{e}\right) \\
& =\left[R_{\tilde{b}, \mathrm{av}}\left(l_{o} T_{e}\right)^{\prime}+\mu_{s}{ }^{2} \pi_{s} \tilde{\mathbf{h}}_{s} \tilde{\mathbf{h}}_{s}^{\dagger}\right]^{-1} \mathbf{r}_{\tilde{x} s, \text { av }}\left(l_{o} T_{e}\right) \\
& =\left[\mathbf{w}_{1}\left(l_{o} T_{e}\right)^{\mathrm{T}}, \mathbf{w}_{1}\left(l_{o} T_{e}\right)^{\dagger}\right]^{\mathrm{T}} \\
& =\left[\frac{\mu_{s} \pi_{s}}{\left(1+\mu_{s}{ }^{2} \pi_{s} \tilde{\mathbf{h}}_{s}^{\dagger} R_{\tilde{b}, \mathrm{av}}\left(l_{o} T_{e}\right)^{\prime-1} \tilde{\mathbf{h}}_{s}\right.}\right] R_{\tilde{b}, \mathrm{av}}\left(l_{o} T_{e}\right)^{\prime-1} \tilde{\mathbf{h}}_{s} .
\end{aligned}
$$

In (24), $R_{\tilde{b}, \text { av }}\left(l_{o} T_{e}\right)^{\prime}$ is defined by (21) with $\tilde{\mathbf{b}}_{v}\left(\left(l_{o} / q+n\right) T\right)^{\prime}$ instead of $\widetilde{\mathbf{x}}_{v}((l / q+n) T)$. The WL SMF is the WL spatial filter which maximizes the output signal-to-interferenceplus-noise ratio (SINR) [17]. Using the previous results, $C_{\mathrm{OPT}-\mathrm{LS}}\left(l_{o} T_{e}\right)$, defined by (22) with $l=l_{o}$, takes the form

$$
C_{\mathrm{OPT}-\mathrm{LS}}\left(l_{o} T_{e}\right)=\frac{\operatorname{SINR}_{y}[\mathrm{OPT}-\mathrm{LS}]}{1+\mathrm{SINR}_{y}[\mathrm{OPT}-\mathrm{LS}]}=\mu_{s} \tilde{\mathbf{w}}\left(l_{o} T_{e}\right)^{\dagger} \tilde{\mathbf{h}}_{s} .
$$

In (25), SINR $_{y}$ [OPT-LS] is the SINR at the output of the WL $\mathrm{SMF}, \widetilde{\mathbf{w}}\left(l_{o} T_{e}\right)$, defined by the ratio between the time-averaged powers, over the training sequence duration, of the considered useful path $i_{0}$ and of the total noise plus other paths at the output of $\tilde{\mathbf{w}}\left(l_{o} T_{e}\right)$. This SINR can be written as

$$
\operatorname{SINR}_{y}[\mathrm{OPT}-\mathrm{LS}]=\mu_{s}^{2} \pi_{s} \widetilde{\mathbf{h}}_{s}^{\dagger} R_{\tilde{b}, \text { av }}\left(l_{o} T_{e}\right)^{\prime-1} \widetilde{\mathbf{h}}_{s}
$$

A similar reasoning can be done for the CONV-LS receiver by replacing $\widetilde{\mathbf{x}}_{v}((l / q+n) T)$ and the WL filter $\hat{\tilde{\mathbf{w}}}\left(l T_{e}\right)$ by $\mathbf{x}_{v}((l / q+n) T)$ and the linear filter $\widehat{\mathbf{w}}\left(l T_{e}\right)=\widehat{R}_{x}\left(l T_{e}\right)^{-1} \widehat{\mathbf{r}}_{x s}\left(l T_{e}\right)$, respectively. Structure of CONV-LS receiver is then depicted at Figure 2 where $y_{v L}((l / q+n) T) \triangleq \widehat{\mathbf{w}}\left(l T_{e}\right)^{\dagger} \mathbf{x}_{v}((l / q+n) T)$, which is a complex quantity, replaces $y_{v \mathrm{WL}}((l / q+n) T)$ appearing in Figure 1. For $l=l_{o}$ and as long as $\mathbf{b}_{T v}((l / q+n) T)^{\prime}$ remains uncorrelated with $s_{v}(n T), \widehat{\mathbf{w}}\left(l T_{e}\right)$ becomes an estimate of the well-known linear SMF, $\mathbf{w}\left(l_{o} T_{e}\right)$, defined by

$$
\begin{aligned}
\mathbf{w}\left(l_{o} T_{e}\right) & \triangleq R_{x, \mathrm{av}}\left(l_{o} T_{e}\right)^{-1} \mathbf{r}_{x s, \mathrm{av}}\left(l_{o} T_{e}\right) \\
& =\left[R_{\mathrm{av}}\left(l_{o} T_{e}\right)^{\prime}+\mu_{s}{ }^{2} \pi_{s} \mathbf{h}_{s} \mathbf{h}_{s}^{\dagger}\right]^{-1} \mathbf{r}_{x s, \mathrm{av}}\left(l_{o} T_{e}\right) \\
& =\left[\frac{\mu_{s} \pi_{s}}{\left(1+\mu_{s}{ }^{2} \pi_{s} \mathbf{h}_{s}^{\dagger} R_{\mathrm{av}}\left(l_{o} T_{e}\right)^{\prime-1} \mathbf{h}_{s}\right)}\right] R_{\mathrm{av}}\left(l_{o} T_{e}\right)^{\prime-1} \mathbf{h}_{s}
\end{aligned}
$$

In (27), $R_{x, \mathrm{av}}\left(l_{o} T_{e}\right)$ and $R_{\mathrm{av}}\left(l_{o} T_{e}\right)^{\prime}$ are defined by (21) with $\mathbf{x}_{v}\left(\left(l_{o} / q+n\right) T\right)$ and $\mathbf{b}_{v}\left(\left(l_{o} / q+n\right) T\right)^{\prime}$ instead of $\tilde{\mathbf{x}}_{v}((l / q+n) T)$, respectively, whereas $\mathbf{r}_{x s \text {,av }}\left(l_{o} T_{e}\right)$ is defined by (20) with $\mathbf{x}_{v}\left(\left(l_{o} / q+n\right) T\right)$ instead of $\tilde{\mathbf{x}}_{v}((l / q+n) T)$. The SMF is the linear spatial filter which maximizes the output signal-to-interference-plus-noise ratio (SINR) [17] 


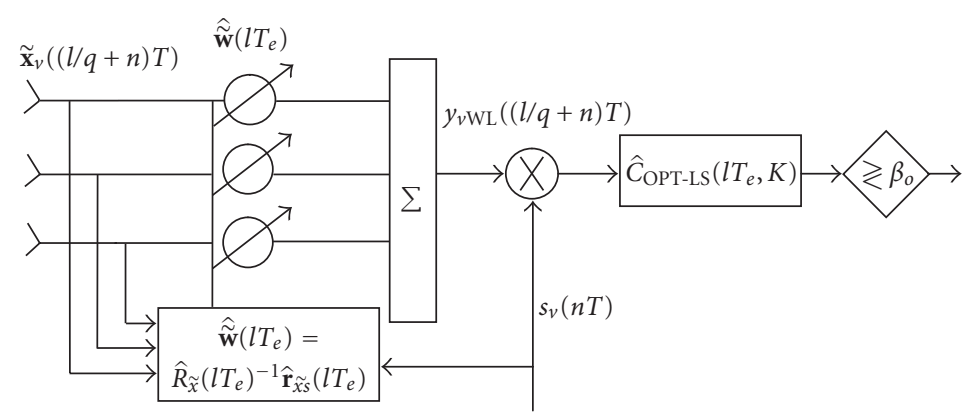

FIgURE 1: Functional scheme of the OPT-LS receiver.

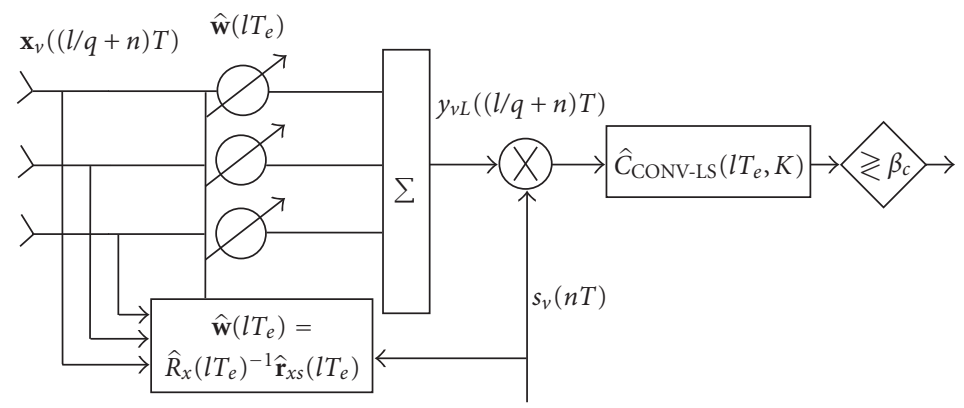

FIGURE 2: Functional scheme of the CONV-LS receiver.

and $C_{\text {CONV-LS }}\left(l_{o} T_{e}\right)$, defined by $(22)$ with $\mathbf{w}\left(l_{o} T_{e}\right)$ instead of $\tilde{\mathbf{w}}\left(l T_{e}\right)$, takes the form

$$
\begin{aligned}
C_{\mathrm{CONV}-\mathrm{LS}}\left(l_{o} T_{e}\right) & =\frac{\mathbf{r}_{x s, \mathrm{av}}\left(l_{o} T_{e}\right)^{\dagger} R_{x, \mathrm{av}}\left(l_{o} T_{e}\right)^{-1} \mathbf{r}_{x s, \mathrm{av}}\left(l_{o} T_{e}\right)}{\pi_{s}} \\
& =\frac{\operatorname{SINR}_{y}[\mathrm{CONV}-\mathrm{LS}]}{1+\operatorname{SINR}_{y}[\mathrm{CONV}-\mathrm{LS}]}=\mu_{s} \mathbf{w}\left(l_{o} T_{e}\right)^{\dagger} \mathbf{h}_{s} .
\end{aligned}
$$

In (28), SINR $_{y}[$ CONV-LS] is the SINR at the output of the $\mathrm{SMF}, \mathbf{w}\left(l_{o} T_{e}\right)$, given by [17]

$$
\operatorname{SINR}_{y}[\mathrm{CONV}-\mathrm{LS}]=\mu_{s}^{2} \pi_{s} \mathbf{h}_{s}^{\dagger} R_{\mathrm{av}}\left(l_{o} T_{e}\right)^{\prime-1} \mathbf{h}_{s} .
$$

Expressions (25) and (28) show that $C_{\mathrm{OPT}-\mathrm{LS}}\left(l_{o} T_{e}\right)$ and $C_{\mathrm{CONV}-\mathrm{LS}}\left(l_{o} T_{e}\right)$ are increasing functions of $\mathrm{SINR}_{y}$ [OPT-LS] and SINR $_{y}[\mathrm{CONV}-\mathrm{LS}]$, respectively, approaching unity for high values of the latter quantities. Note that for a circular total noise, $\operatorname{SINR}_{y}[\mathrm{OPT}-\mathrm{LS}]=2 \mathrm{SINR}_{y}[\mathrm{CONV}$-LS $]$. In the presence of rectilinear interferences, the WL SMF (24) is shown in [17] to correspond to a classical SMF but for a virtual array of $2 \mathrm{~N}$ sensors with phase diversity in addition to space, angular, and/or polarization diversities of the true array of $N$ sensors. The SMF (27) discriminates the useful signal and interferences by the direction of arrival (DOA) and/or polarization (if $N>1$ ) and is able to reject up to $N-1$ interferences from an array of $N$ sensors. The WL SMF (24) discriminates the sources by DOA, polarization (if $N>1$ ) and phase, and is thus able to reject up to $2 N-1$ rectilinear interferences from an array of $N$ sensors [17]. It allows in particular the rejection of one rectilinear interference from one antenna, hence the single antenna interference cancellation (SAIC) concept described in detail in [17]. In these conditions, the correlation operation between the training sequence, $s_{v}(n T)$, and the output, $y_{v \mathrm{WL}}\left(\left(l_{o} / q+n\right) T\right)$, of $\hat{\tilde{\mathbf{w}}}\left(l_{o} T_{e}\right)$, allows the generation of a correlation maxima from a limited number of useful symbols $K$, whose minimum value has to increase when the asymptotic output SINR decreases (see next section).

\subsection{Performance}

As it has been discussed in Sections 2.3 and 3, the synchronization problem can be seen either as an estimation or as a detection problem. Moreover, when the number $M$ of useful paths is not known a priori, a threshold is required to limit the FAR. For this reason, for each useful path $i_{0}$, performances of OPT-LS and CONV-LS receivers are computed in this paper in terms of detection probability of the optimal delay $l_{o} T_{e}$ for a given FAR. The FAR corresponds to the probability that $\hat{C}_{\mathrm{OPT}-\mathrm{LS}}\left(l_{o} T_{e}, K\right)$ (resp., $\left.\hat{C}_{\mathrm{CONV}-\mathrm{LS}}\left(l_{o} T_{e}, K\right)\right)$ gets beyond the thresholds, $\beta_{o}$ (resp., $\beta_{c}$ ), under H0, where, for a given FAR, $\beta_{o}$ and $\beta_{c}$ are functions of $N, K$, the number and the level of rectilinear interferences into $\mathbf{b}_{T v}\left(\left(l_{o} / q+\right.\right.$ $n) T)$. Moreover, the probability of detection of the delay $l_{o} T_{e}$, noted $P_{d}$, is the probability that $\hat{C}_{\mathrm{OPT}-\mathrm{LS}}\left(l_{o} T_{e}, K\right)$ (resp., $\left.\hat{C}_{\mathrm{CONV}-\mathrm{LS}}\left(l_{o} T_{e}, K\right)\right)$ gets beyond the thresholds, $\beta_{o}$ (resp., $\beta_{c}$ ). The analytical computation of $P_{d}$ for a given FAR has been done in $[8,10]$ for the CONV-LS receiver but under the assumption of orthogonal training sequences and Gaussian and circular total noise. However, in the present paper, the training sequences are not assumed to be orthogonal and the 
total noise is not Gaussian and not circular in the presence of rectilinear interferences. For these reasons, the results of $[8,10]$ are no longer valid for rectilinear sources whereas the analytical computation of the true $P_{d}$ for OPT-LS and CONV-LS receivers seems to be a difficult task which will be investigated elsewhere. Nevertheless, for not too small values of $K$, we deduce from the central limit theorem that the contribution of the total noise in (18) is not far from being Gaussian. This means that the detection probability $P_{d}$ is not far from being related to the SINR, noted $\widehat{\operatorname{SINR}}_{c}$ [OPT-LS] $(K)$, at the output of the correlation between the training sequence $s_{v}(n T)$ and the output $y_{v \mathrm{WL}}\left(\left(l_{o} / q+n\right) T\right)$. Using (3) into (18) for $l=l_{o}$, we obtain

$$
\begin{aligned}
\hat{C}_{\mathrm{OPT}-\mathrm{LS}} & \left(l_{o} T_{e}, K\right) \\
= & \mu_{s} \hat{\tilde{\mathbf{w}}}\left(l_{o} T_{e}\right)^{\dagger} \tilde{\mathbf{h}}_{s} \\
& +\left(\frac{1}{K \pi_{s}}\right) \hat{\tilde{\mathbf{w}}}\left(l_{o} T_{e}\right)^{\dagger} \sum_{n=0}^{K-1} \tilde{\mathbf{b}}_{T v}\left(\left(\frac{l_{o}}{q}+n\right) T\right)^{\prime} \boldsymbol{s}_{v}(n T) .
\end{aligned}
$$

To go further in the computation of the OPT-LS receiver performance, we assume that assumptions (A1ter), (A2bis), and (A6bis) are verified, where these assumptions are defined by:

(A1ter) the samples $\tilde{\mathbf{b}}_{T v}\left(\left(l_{o} / q+n\right) T\right)^{\prime}, 0 \leq n \leq K-1$, are uncorrelated to each other,

(A2bis) the matrices $R\left(\left(l_{o} / q+n\right) T\right)^{\prime}$ and $C\left(\left(l_{o} / q+n\right) T\right)^{\prime}$ do not depend on the symbol indice $n$,

(A6bis) the samples $\mathbf{b}_{T v}\left(\left(l_{o} / q+n\right) T\right)^{\prime}$ and $s_{v}(m T), 0 \leq n$, $m \leq K-1$, are statistically independent.

From these assumptions and using the fact that the filter $\hat{\tilde{\mathbf{w}}}\left(l_{o} T_{e}\right)$ is not random over the training sequence duration (although it is random over several training sequences durations), the $\widehat{\operatorname{SINR}}_{c}[\mathrm{OPT}-\mathrm{LS}](K)$, defined by the ratio between the expected value of the square modulus of the two terms of the right-hand side of expression (30), is given by

$$
\widehat{\operatorname{SINR}}_{c}[\mathrm{OPT}-\mathrm{LS}](K)=K \widehat{\operatorname{SINR}}_{y}[\mathrm{OPT}-\mathrm{LS}](K) .
$$

In (31), $\widehat{\operatorname{SINR}}_{y}[\mathrm{OPT}-\mathrm{LS}](K)$ is the SINR at the output, $y_{v \mathrm{WL}}\left(\left(l_{o} / q+n\right) T\right)$, of the WL filter $\hat{\tilde{\mathbf{w}}}\left(l_{o} T_{e}\right)$, given, under (A2bis), by

$$
\widehat{\operatorname{SINR}}_{y}[\operatorname{OPT}-\mathrm{LS}](K)=\frac{\mu_{s}^{2} \pi_{s}\left|\hat{\tilde{\mathbf{w}}}\left(l_{o} T_{e}\right)^{\dagger} \widetilde{\mathbf{h}}_{s}\right|^{2}}{\hat{\tilde{\mathbf{w}}}\left(l_{o} T_{e}\right)^{\dagger} R_{\widetilde{b}}\left(l_{o} T_{e}\right)^{\prime} \hat{\tilde{\mathbf{w}}}\left(l_{o} T_{e}\right)},
$$

where $R_{\widetilde{b}}\left(l_{o} T_{e}\right)^{\prime}$ is defined by (17) with $\tilde{\mathbf{b}}_{T v}\left(\left(l_{o} / q+n\right) T\right)^{\prime}$ instead of $\tilde{\mathbf{b}}_{T v}\left(\left(l_{o} / q+n\right) T\right)$. A similar reasoning can be done for the CONV-LS receiver under the same assumptions, by replacing the real output $y_{v \mathrm{WL}}\left(\left(l_{o} / q+n\right) T\right)$ by the real quantity $z_{v L}\left(\left(l_{o} / q+n\right) T\right) \triangleq \operatorname{Re}\left[y_{v L}\left(\left(l_{o} / q+n\right) T\right)\right] \triangleq$ $\operatorname{Re}\left[\widehat{\mathbf{w}}\left(l_{o} T_{e}\right)^{\dagger} \mathbf{x}_{v}\left(\left(l_{o} / q+n\right) T\right)\right]$. Noting $\widehat{\operatorname{SINR}}_{c}[\mathrm{CONV}-\mathrm{LS}](K)$, the SINR at the output of the correlation between the training sequence $s_{v}(n T)$ and $z_{v L}\left(\left(l_{o} / q+n\right) T\right)$, we obtain

$$
\widehat{\operatorname{SINR}}_{c}[\mathrm{CONV}-\mathrm{LS}](K)=K \widehat{\operatorname{SINR}}_{z}[\mathrm{CONV}-\mathrm{LS}](K),
$$

where $\widehat{\operatorname{SINR}}_{z}[\mathrm{CONV}-\mathrm{LS}](K)$ is the SINR in the output $z_{v L}\left(\left(l_{o} / q+n\right) T\right)$, given, under (A2bis), by

$\widehat{\operatorname{SINR}}_{z}[\mathrm{CONV}-\mathrm{LS}](K)$

$$
=\frac{2 \mu_{s}^{2} \pi_{s}\left|\operatorname{Re}\left[\widehat{\mathbf{w}}\left(l_{o} T_{e}\right)^{\dagger} \mathbf{h}_{s}\right]\right|^{2}}{\widehat{\mathbf{w}}\left(l_{o} T_{e}\right)^{\dagger} R\left(l_{o} T_{e}\right)^{\prime} \hat{\mathbf{w}}\left(l_{o} T_{e}\right)+\operatorname{Re}\left[\widehat{\mathbf{w}}\left(l_{o} T_{e}\right)^{\dagger} C\left(l_{o} T_{e}\right)^{\prime} \hat{\mathbf{w}}\left(l_{o} T_{e}\right)^{*}\right]} .
$$

Expressions (31) and (33) show that $\widehat{\operatorname{SINR}}_{c}[\mathrm{OPT}-\mathrm{LS}](K)$ and $\widehat{\operatorname{SINR}}_{c}[\mathrm{CONV}-\mathrm{LS}](K)$, and thus the detection performance of the associated receivers, increase with the number of symbols, $K$, of the training sequence and with the SINR, $\widehat{\operatorname{SINR}}_{y}[\mathrm{OPT}-\mathrm{LS}](K)$ and $\widehat{\operatorname{SINR}}_{z}[\mathrm{CONV}-\mathrm{LS}](K)$, in the real part of the output of the filters $\hat{\tilde{\mathbf{w}}}\left(l_{o} T_{e}\right)$ and $\hat{\mathbf{w}}\left(l_{o} T_{e}\right)$, respectively.

Under (A2bis), as the number of symbols, $K$, of the training sequence becomes infinite, $\widehat{\operatorname{SINR}}_{y}[\mathrm{OPT}-\mathrm{LS}](K)$ and $\widehat{\operatorname{SINR}}_{z}[\mathrm{CONV}-\mathrm{LS}](K)$ tend toward the quantities $\operatorname{SINR}_{y} \times$ [OPT-LS] $\triangleq \lim _{K \rightarrow \infty} \widehat{\operatorname{SINR}}_{y}[\mathrm{OPT}-\mathrm{LS}](K)$, defined by (26), and $\operatorname{SINR}_{z}[\mathrm{CONV}-\mathrm{LS}] \triangleq \lim _{K \rightarrow \infty} \widehat{\operatorname{SINR}}_{z}[\mathrm{CONV}-\mathrm{LS}](K)$, defined by

$$
\begin{aligned}
& \operatorname{SINR}_{z}[\mathrm{CONV}-\mathrm{LS}]= \\
& \frac{2 \mu_{s}{ }^{2} \pi_{s} \mathbf{h}_{s}^{\dagger} R\left(l_{o} T_{e}\right)^{\prime-1} \mathbf{h}_{s}}{1+\operatorname{Re}\left[\mathbf{h}_{s}^{\dagger} R\left(l_{o} T_{e}\right)^{\prime-1} C\left(l_{o} T_{e}\right)^{\prime} R\left(l_{o} T_{e}\right)^{\prime-1 *} \mathbf{h}_{s}^{*} / \mathbf{h}_{s}^{\dagger} R\left(l_{o} T_{e}\right)^{\prime-1} \mathbf{h}_{s}\right]}
\end{aligned}
$$

respectively. Note that $\mathrm{SINR}_{z}[\mathrm{CONV}-\mathrm{LS}]$ corresponds to $2 \mathrm{SINR}_{y}[\mathrm{CONV}-\mathrm{LS}]$ and to $\mathrm{SINR}_{y}[\mathrm{OPT}-\mathrm{LS}]$ for SO circular vectors $\mathbf{b}_{T v}\left(\left(l_{o} / q+n\right) T\right)^{\prime}\left(C\left(l_{o} T_{e}\right)^{\prime}=0\right)$. Noting $\widehat{\operatorname{SINR}}_{y} \times$ $[\mathrm{CONV}-\mathrm{LS}](K)$, the SINR at the output, $y_{v L}\left(\left(l_{o} / q+n\right) T\right)$, of the filter $\widehat{\mathbf{w}}\left(l_{o} T_{e}\right)$, it has been shown in [35], under an assumption of stationary and Gaussian observations, that for a given value of $\operatorname{SINR}_{y}[\mathrm{CONV}-\mathrm{LS}]$, it exists a number $K_{c y}$, increasing with $1 / \mathrm{SINR}_{y}[\mathrm{CONV}-\mathrm{LS}]$ such that $\widehat{\operatorname{SINR}}_{y}[\mathrm{CONV}-\mathrm{LS}](K) \approx \mathrm{SINR}_{y}[\mathrm{CONV}-\mathrm{LS}]$ for $K>K_{c y}$. Results of Table 1, built from empirical computer simulations, show that a similar result seems to also exist in the presence of rectilinear interferences and seems to also hold for $\widehat{\operatorname{SINR}}_{z}[\mathrm{CONV}-\mathrm{LS}](K)$ and $\widehat{\operatorname{SINR}}_{y}[\mathrm{OPT}-\mathrm{LS}](K)$. In other words, it seems to exist numbers $K_{o y}$ and $K_{c z}$, increasing with $1 / \mathrm{SINR}_{y}[\mathrm{OPT}-\mathrm{LS}]$ and $1 / \mathrm{SINR}_{z}[\mathrm{CONV}-\mathrm{LS}]$, respectively, such that

$$
\begin{aligned}
& \widehat{\operatorname{SINR}}_{c}[\mathrm{CONV}-\mathrm{LS}](K) \approx K \mathrm{SINR}_{z}[\mathrm{CONV}-\mathrm{LS}] \text { for } K>K_{c z} \text {, } \\
& \widehat{\operatorname{SINR}}_{c}[\mathrm{OPT}-\mathrm{LS}](K) \approx K \mathrm{SINR}_{y}[\mathrm{OPT}-\mathrm{LS}] \text { for } K>K_{o y} \text {, }
\end{aligned}
$$

which allows a simple description of the approximated performance of both the CONV-LS and OPT-LS receivers from $K$ and expressions (35) and (26), respectively, provided that $K>K_{c z}$ and $K>K_{o y}$, respectively. Some insights about the values of $K_{c y}, K_{c z}$ and $K_{o y}$ are given in Section 4 . 


\section{PERFORMANCE OF CONV-LS AND OPT-LS RECEIVERS IN THE PRESENCE OF A BPSK SIGNAL AND ONE RECTILINEAR INTERFERENCE}

\subsection{Total noise model}

To quantify the performance of the previous receivers for the detection of the useful path $i_{0}$, we assume that the vector $\mathbf{b}_{T v}\left(k T_{e}\right)^{\prime}$ is composed of one rectilinear interference, with the same waveform as the useful path $i_{0}$, and a background noise. This interference, which is assumed to be uncorrelated with the useful path $i_{0}$, may be generated by the network itself or corresponds to a decorrelated useful path different from $i_{0}$. Under this assumption, the vector $\mathbf{b}_{T v}\left(k T_{e}\right)^{\prime}$ can be written as

$$
\mathbf{b}_{T v}\left(k T_{e}\right)^{\prime} \approx j_{1 v}\left(k T_{e}\right) \mathbf{h}_{1}+\mathbf{b}_{v}\left(k T_{e}\right),
$$

where $\mathbf{b}_{v}\left(k T_{e}\right)$ is the sampled background noise vector, assumed zero-mean, stationary, Gaussian, SO circular and spatially white, $\mathbf{h}_{1}$ is the channel impulse response vector of the interference and $j_{1 v}\left(k T_{e}\right)$ is the sampled complex envelope of the interference after the matched filtering operation. Moreover, the matrices $R\left(k T_{e}\right)^{\prime}$ and $C\left(k T_{e}\right)^{\prime}$, defined in Section 2.2, can be written as

$$
\begin{gathered}
R\left(k T_{e}\right)^{\prime} \approx \pi_{1}\left(k T_{e}\right) \mathbf{h}_{1} \mathbf{h}_{1}^{\dagger}+\eta_{2} I, \\
C\left(k T_{e}\right)^{\prime} \approx \pi_{1}\left(k T_{e}\right) \mathbf{h}_{1} \mathbf{h}_{1}^{\mathrm{T}} .
\end{gathered}
$$

In the previous expressions, $\eta_{2}$ is the mean power of the background noise per sensor, $I$ is the $(N \times N)$ identity matrix, and $\pi_{1}\left(k T_{e}\right) \triangleq \mathrm{E}\left[\left|j_{1 v}\left(k T_{e}\right)\right|^{2}\right]$ is the power of the interference at the output of the filter $v(-t)^{*}$ received by an omnidirectional sensor for a free space propagation. Finally, we define the spatial correlation coefficient between the interference and the useful signal, $\alpha_{1 s}$, such that $0 \leq\left|\alpha_{1 s}\right| \leq 1$, by

$$
\alpha_{1 s} \triangleq \frac{\mathbf{h}_{1}^{\dagger} \mathbf{h}_{s}}{\left(\mathbf{h}_{1}^{\dagger} \mathbf{h}_{1}\right)^{1 / 2}\left(\mathbf{h}_{s}^{\dagger} \mathbf{h}_{s}\right)^{1 / 2}} \triangleq\left|\alpha_{1 s}\right| e^{-j \psi}
$$

where $\psi$ is the phase of $\mathbf{h}_{s}^{\dagger} \mathbf{h}_{1}$.

\subsection{Output SINR computation}

The computation of the quantities $\operatorname{SINR}_{z}[\mathrm{CONV}-\mathrm{LS}]$ and SINR $_{y}[$ OPT-LS] in the presence of one rectilinear interference have been done in [17] for demodulation purposes. For this reason, we just recall the main results of [17] to show off both the interests of OPT-LS receiver and the limitations of CONV-LS receiver in the presence of one rectilinear interference.

When there is no spatial discrimination between the sources, that is, when $\left|\alpha_{1 s}\right|=1$, which occurs in particular for a mono-sensor reception $(N=1), \operatorname{SINR}_{z}[\mathrm{CONV}-\mathrm{LS}]$
TABLE 1: $K_{c y}, K_{c z}$, and $K_{o z}$ as a function of $N$ and $\operatorname{SINR}_{y}$ [CONV-LS], $\operatorname{SINR}_{z}[\mathrm{CONV}-\mathrm{LS}]$, and $\operatorname{SINR}_{z}[\mathrm{OPT}-\mathrm{LS}]$, respectively, $|\operatorname{RMS}[\rho]|=$ $1 \mathrm{~dB}$, BPSK signals.

\begin{tabular}{c|c|c}
\hline & $N=1$ & $N>1$ \\
\hline$K_{c y}$ & 1 & $5 N-6+(4 N-5.8) / \mathrm{SINR}_{c y}$ \\
\hline$K_{c z}$ & $2+63.3 / \mathrm{SINR}_{c z}$ & $5 N-6+(8.2 N-1) \mathrm{SINR}_{c z}$ \\
\hline$K_{o z}$ & \multicolumn{2}{|c}{$10 N-6+(7.8 N-4.8) / \mathrm{SINR}_{o z}$} \\
\hline
\end{tabular}

and $\mathrm{SINR}_{y}[\mathrm{OPT}-\mathrm{LS}]$ can be written, under the assumptions of the previous sections, as

$$
\begin{gathered}
\operatorname{SINR}_{z}[\mathrm{CONV}-\mathrm{LS}]=\frac{2 \varepsilon_{s}}{1+2 \varepsilon_{1} \cos ^{2} \psi} ; \quad\left|\alpha_{1 s}\right|=1, \\
\operatorname{SINR}_{y}[\mathrm{OPT}-\mathrm{LS}]=2 \varepsilon_{s}\left[1-\frac{2 \varepsilon_{1}}{1+2 \varepsilon_{1}} \cos ^{2} \psi\right] ; \quad\left|\alpha_{1 s}\right|=1,
\end{gathered}
$$

where $\varepsilon_{s} \triangleq\left(\mathbf{h}_{s}^{\dagger} \mathbf{h}_{s}\right) \mu_{s}{ }^{2} \pi_{s} / \eta_{2}$ and $\varepsilon_{1} \triangleq\left(\mathbf{h}_{1}^{\dagger} \mathbf{h}_{1}\right) \pi_{1}\left(l_{0} T_{e}\right) / \eta_{2}$. When $\psi=\pi / 2+k \pi$, that is, when the useful path $i_{0}$ and interference are in quadrature, the previous expressions are equivalent, maximal, and equal to $2 \varepsilon_{s}$, which proves a complete interference rejection both in the real part of the output of the SMF, $\mathbf{w}\left(l_{o} T_{e}\right)$, and at the output of the WL SMF, $\widetilde{\mathbf{w}}\left(l_{o} T_{e}\right)$. Otherwise, as $\varepsilon_{1}$ becomes infinitely large, $\operatorname{SINR}_{z}$ [CONV-LS] decreases to zero, which proves the absence of interference rejection by the SMF, and thus, from (36), the difficulty to detect the useful path $i_{0}$ in the presence of a strong interference from the CONV-LS receiver for small values of $K$. However, for large values of $\varepsilon_{1}, \mathrm{SINR}_{y}[\mathrm{OPT}-\mathrm{LS}]$ can be approximated by

$$
\begin{gathered}
\operatorname{SINR}_{y}[\mathrm{OPT}-\mathrm{LS}] \approx 2 \varepsilon_{s}\left[1-\cos ^{2} \psi\right] ; \\
\varepsilon_{1} \gg 1,\left|\alpha_{1 s}\right|=1, \psi \neq 0+k \pi
\end{gathered}
$$

which becomes independent of $\varepsilon_{1}$, which is solely controlled by quantities $2 \varepsilon_{s}$ and $\cos ^{2} \psi$ and which proves an interference rejection by the WL SMF, depending on the parameter $\psi$, hence the SAIC capability as long as $\psi \neq 0+k \pi$, that is, as long as there is a phase discrimination between useful path $i_{0}$ and interference. This proves, from (37), the potential capability of the OPT-LS receiver to detect the useful path $i_{0}$ in the presence of a strong rectilinear interference even for small values of $K$ and despite the fact that $\left|\alpha_{1 s}\right|=1$.

When there is a spatial discrimination between useful signal and interference $\left(\left|\alpha_{1 s}\right| \neq 1\right)$, which occurs in most situations for $N>1$, as $\varepsilon_{1}$ becomes infinitely large, we obtain

$$
\begin{aligned}
& \operatorname{SINR}_{z}[\mathrm{CONV}-\mathrm{LS}] \approx 2 \varepsilon_{s}\left[1-\left|\alpha_{1 s}\right|^{2}\right] ; \quad \varepsilon_{1} \gg 1,\left|\alpha_{1 s}\right| \neq 1, \\
& \operatorname{SINR}_{y}[\mathrm{OPT}-\mathrm{LS}] \approx 2 \varepsilon_{s}\left[1-\left|\alpha_{1 s}\right|^{2} \cos ^{2} \psi\right] ; \\
& \varepsilon_{1} \gg 1,\left|\alpha_{1 s}\right| \neq 1 .
\end{aligned}
$$

These expressions are maximal, equal to $2 \varepsilon_{s}$ and the interference is completely rejected in both cases when $\left|\alpha_{1 s}\right|=0$, that 
is, when the propagation channel vectors of the interference and the useful path $i_{0}$ are orthogonal. Otherwise, these expressions remain independent of $\varepsilon_{1}$ and are solely controlled by $2 \varepsilon_{s}$, by the square modulus of the spatial correlation coefficient between useful $i_{0}$ and interference and (for OPT-LS receiver) by the phase difference between the sources. These results prove an interference rejection by both the SMF and the WL SMF, but while this rejection is based on a spatial discrimination only in the first case, it is based on both a spatial and a phase discrimination in the second case. This allows in particular to reject an interference having the same direction of arrival and the same polarization as the useful path $i_{0}$, which finally allows better synchronization performance in the presence of rectilinear interferences from the OPT-LS receiver.

\subsection{Computer simulations}

We first give some insights into the values of $K_{c y}, K_{c z}$, and $K_{o y}$ introduced in Section 3.4. Then, we illustrate some variations of the sufficient statistics $\hat{C}_{\mathrm{CONV}-\mathrm{LS}}\left(l T_{e}, K\right)$ and $\hat{C}_{\mathrm{OPT}-\mathrm{LS}}\left(l T_{e}, K\right)$ and finally, we compute and illustrate the variations of the probability of nondetection of the optimal delay, $l_{o} T_{e}$, by the CONV-LS and OPT-LS receivers, for a given FAR.

\subsubsection{Some insights into the values of $K_{c y}, K_{c z}$ and $K_{o y}$}

To give some insights into the values of $K_{c y}, K_{c z}$ and $K_{o y}$, we introduce the quantities

$$
\begin{aligned}
& \rho_{c y}(K) \triangleq \frac{\widehat{\operatorname{SINR}}_{y}[\mathrm{CONV}-\mathrm{LS}](K)}{\operatorname{SINR}_{y}[\mathrm{CONV}-\mathrm{LS}]}, \\
& \rho_{c z}(K) \triangleq \frac{\widehat{\operatorname{SINR}}_{z}[\mathrm{CONV}-\mathrm{LS}](K)}{\mathrm{SINR}_{z}[\mathrm{CONV}-\mathrm{LS}]}, \\
& \rho_{o y}(K) \triangleq \frac{\widehat{\operatorname{SINR}}_{y}[\mathrm{OPT}-\mathrm{LS}](K)}{\operatorname{SINR}_{y}[\mathrm{OPT}-\mathrm{LS}]}
\end{aligned}
$$

Note that $0 \leq \rho_{c z}(K) \leq 1$ for circular vectors $\mathbf{b}_{T v}\left(k T_{e}\right)^{\prime}$ only, whereas $0 \leq \rho_{c y}(K) \leq 1$ and $0 \leq \rho_{o y}(K) \leq 1$ in all cases. For given scenario of useful signal and total noise, for a given array of $N$ sensors and a given number of symbols, $K$, of the training sequence, we compute $M$ independent realizations of the filters $\hat{\mathbf{w}}\left(l_{o} T_{e}\right)$, and $\hat{\tilde{\mathbf{w}}}\left(l_{o} T_{e}\right)$ and then $M$ independent realizations of the quantities $\widehat{\operatorname{SINR}}_{y}[\mathrm{CONV}-\mathrm{LS}](K)$, $\widehat{\operatorname{SINR}}_{z}[\mathrm{CONV}-\mathrm{LS}](K)$ and $\widehat{\operatorname{SINR}}_{y}[\mathrm{OPT}-\mathrm{LS}](K)$. From these $M$ independent realizations and for a given ratio $\rho_{v u}(K)(v=$ $c$ or $o, u=y$ or $z$ ) we compute an estimate, $\widehat{\operatorname{RMS}}\left[\rho_{v u}(K)\right]$, of the root mean square (RMS) value of $\rho_{v u}(K), \operatorname{RMS}\left[\rho_{v u}(K)\right]$, defined by

$$
\widehat{\operatorname{RMS}}\left[\rho_{v u}(K)\right] \triangleq\left[\frac{1}{M} \sum_{m=1}^{M} \rho_{v u, m}(K)^{2}\right]^{1 / 2},
$$

where $\rho_{v u, m}(K)$ is the realization $m$ of $\rho_{v u}(K)$. Considering that $K_{c y}, K_{c z}$, and $K_{o y}$ correspond to the number of training symbols $K$ above which $\left|10 \log _{10}\left(\widehat{\operatorname{RMS}}\left[\rho_{c y}(K)\right]\right)\right|$, $\left|10 \log _{10}\left(\widehat{\operatorname{RMS}}\left[\rho_{c z}(K)\right]\right)\right|$, and $\left|10 \log _{10}\left(\widehat{\operatorname{RMS}}\left[\rho_{o y}(K)\right]\right)\right|$, estimated from $M=100000$ realizations, are below $1 \mathrm{~dB}$, respectively, numerous simulations allow to empirically predict, for BPSK signals, analytical expressions of $K_{c y}, K_{c z}$, and $K_{o y}$ as a function of $N$ and the associated asymptotic output SINR. These expressions are summarized in Table 1 and have the same structure as those introduced by Monzingo and Miller [35] for Gaussian observations. Note that when the number of interferences $P$ becomes such that $P \geq N$, expressions related to $K_{c z}$ in Table 1 may be no longer valid. Otherwise, note that for values of $\operatorname{SINR}_{y}[\mathrm{CONV}-\mathrm{LS}]\left(\mathrm{SINR}_{c y}\right)$, $\mathrm{SINR}_{z}[\mathrm{CONV}-\mathrm{LS}]\left(\mathrm{SINR}_{c z}\right)$, and $\mathrm{SINR}_{y}[\mathrm{OPT}-\mathrm{LS}]\left(\mathrm{SINR}_{o y}\right)$ equal to $10 \mathrm{~dB}, K_{c y} \approx 5.4 N-6.6(N>1), K_{c z} \approx 5.8 N-6.1$ $(N>1)$ and $8.33(N=1)$ and $K_{o z} \approx 10.8 N-6.5$. These results show off in particular that (36) and (37) are approximately valid from a very limited number of training symbols for small values of $N$. Besides, for $\operatorname{SINR}_{z}[\mathrm{OPT}-\mathrm{LS}]=0 \mathrm{~dB}$, $K_{o z} \approx 17.8 N-10.8$, which gives $K_{o z} \approx 7$ for $N=1, K_{o z} \approx 25$ for $N=2$ and which remains very weak values.

\subsubsection{Variations of $\widehat{C}_{C O N V-L S}\left(l T_{e}, K\right)$ and $\widehat{C}_{O P T-L S}\left(l T_{e}, K\right)$}

To illustrate the variations of $\hat{C}_{\mathrm{CONV}-\mathrm{LS}}\left(l T_{e}, K\right)$ and $\hat{C}_{\mathrm{OPT} \text {-LS }} \times$ $\left(l T_{e}, K\right)$, we consider a mono-sensor reception $(N=1)$ and we assume that the useful BPSK path $i_{0}$, received with a SNR equal to $5 \mathrm{~dB}$, is perturbed by one BPSK interference having the same pulse-shaped filter and the same symbol rate and with an INR equal to $20 \mathrm{~dB}$. The phase difference $\psi$ between the interference and the useful path $i_{0}$ is equal to $\pi / 4$. The training sequence is assumed to contain $K=64$ symbols and the symbol duration $T$ is such that $T=2 T_{e}$. To simplify the simulation, the optimal delay, $\tau_{i 0}$, is chosen to correspond to a multiple of the sample period, $\tau_{i 0}=l_{o} T_{e}$, such that $l_{o}=139$ on Figure 3(a). Under these assumptions, Figure 3(a) shows the variations of $\hat{C}_{\mathrm{CONV}-\mathrm{LS}}\left(l T_{e}, K\right)$ and $\hat{C}_{\mathrm{OPT} \text {-LS }}\left(l T_{e}, K\right)$, respectively, as a function of the delay $l T_{e}$, jointly with the threshold, $\beta_{c}$ and $\beta_{o}$, associated with these two receivers, respectively, for a FAR equal to 0.001 . Note the nondetection of the optimal delay $l_{o} T_{e}$ from the conventional receiver due to a poor value of $\widehat{\operatorname{SINR}}_{z}[\mathrm{CONV}-\mathrm{LS}](K)$ equal to $-15 \mathrm{~dB}$ and the good detection of this delay from the optimal receiver due to a better value of $\widehat{\operatorname{SINR}}_{z}$ [OPT-LS] $(K)$ equal to $4.7 \mathrm{~dB}$. To complete these results, we consider the previous scenario but where the phase difference $\psi$ is now an adjustable parameter. In these conditions, Figure 3(b) shows the variations of $\hat{C}_{\text {CONV-LS }}\left(l_{o} T_{e}, K\right)$ and $\hat{C}_{\text {OPT-LS }}\left(l_{o} T_{e}, K\right)$ as a function of $\psi$, jointly with the threshold, $\beta_{c}$ and $\beta_{o}$, associated with these two receivers, respectively, for a FAR equal to 0.001 . Note the weak value of $\hat{C}_{\mathrm{CONV}-\mathrm{LS}}\left(l_{o} T_{e}, K\right)$, almost always below the threshold, whatever the parameter $\psi$, preventing the detection of the useful path $i_{0}$ from the conventional receiver in most situations. Note also the values of $\hat{C}_{\mathrm{OPT}-\mathrm{LS}}\left(l_{o} T_{e}, K\right)$ beyond the threshold as soon as the phase difference $\psi$ is not too low. This allows in most cases the detection of the useful 


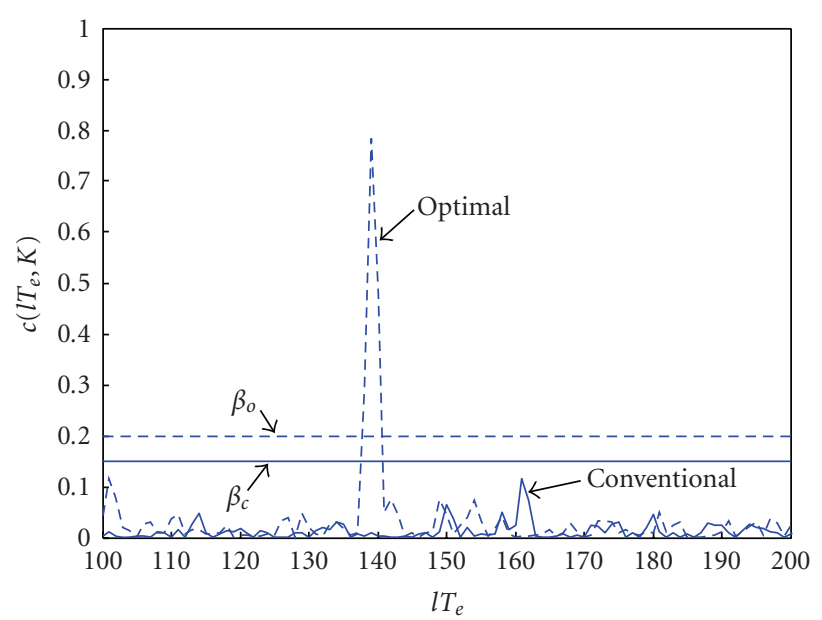

(a)

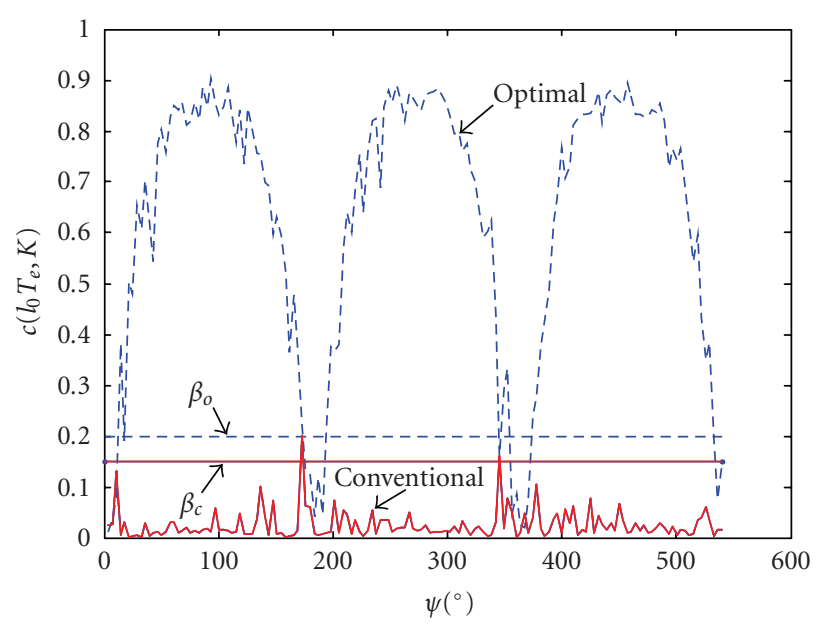

(b)

Figure 3:Variations of $\hat{C}_{\mathrm{CONV}-\mathrm{LS}}\left(l T_{e}, K\right)$ and $\hat{C}_{\mathrm{OPT}-\mathrm{LS}}\left(l T_{e}, K\right)$ as a function of $l T_{e}$ (a), variations of $\hat{C}_{\mathrm{CONV}-\mathrm{LS}}\left(l_{o} T_{e}, K\right)$ and $\hat{C}_{\text {OPT-LS }}\left(l_{o} T_{e}, K\right)$ as a function of $\psi(\mathrm{b}), K=64, T=2 T_{e}$, one interference, $N=1, \pi_{s} / \eta_{2}=5 \mathrm{~dB}$, INR $=20 \mathrm{~dB}, \psi=\pi / 4, \mathrm{FAR}=0.001$.

signal $i_{0}$ in the presence of a strong rectilinear interference from the optimal receiver even from $N=1$ sensor.

\subsubsection{Probability of nondetection for a given FAR}

To quantify the performance of CONV-LS and OPT-LS receivers, we now consider a burst radio communication link for which a training sequence of $K=64$ symbols is transmitted at each burst. The BPSK useful path $i_{0}$ is assumed to be corrupted by a BPSK interference with the same waveform and whose INR is always $20 \mathrm{~dB}$ above the SNR. Note that the interference can be a true interference generated by the network itself or a decorrelated useful path different from $i_{0}$. The array is an ULA of $N$ sensors. The phase and DOA of both the useful path $i_{0}$ and interference are independent random variables, uniformly distributed on $[0,2 \pi]$, and are assumed to change randomly at each burst. The performance

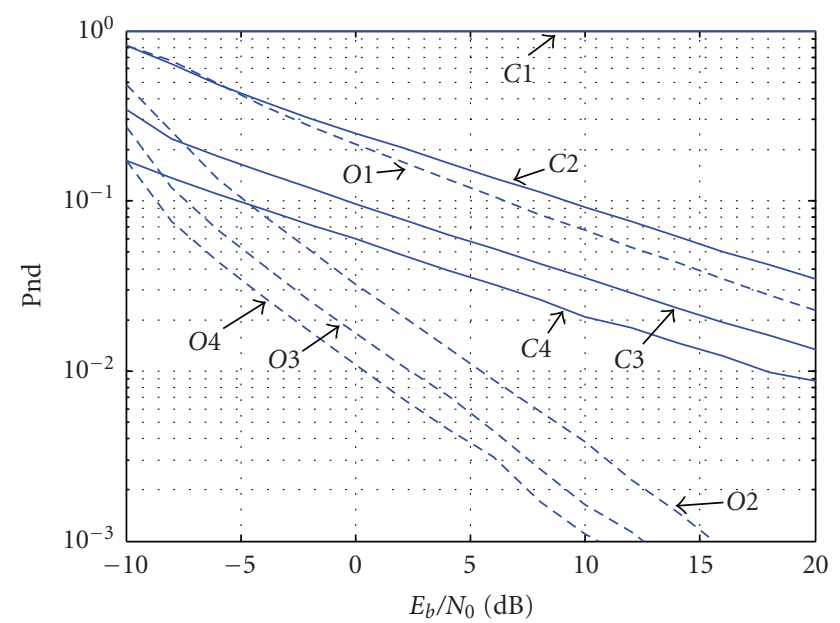

(a)

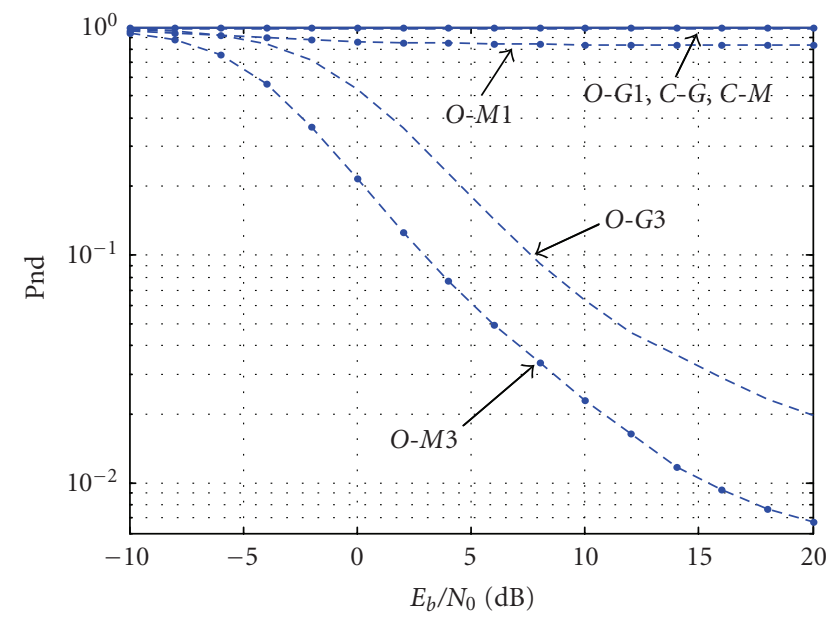

(b)

FIgure 4: Probability of nondetection of CONV-LS (C) and OPTLS $(\mathrm{O})$ receivers as a function of SNR, $K=64, T=2 T_{e}$, one interference, $\mathrm{INR}=\mathrm{SNR}+20 \mathrm{~dB}$, phase, DOA and delay random, FAR $=0.001,100000$ realizations, BPSK and $N=1,2,3,4$ (a), MSK (M), $\operatorname{GMSK}(\mathrm{G}), N=1, L=1,3$ (b).

are evaluated over 100000 bursts. Under these assumptions, Figure 4(a) shows the probability of nondetection of the optimal delay $l_{o} T_{e}$ by the CONV-LS (C) and OPT-LS (O) receivers as a function of the input SNR, $\mu_{s}^{2} \pi_{s} / \eta_{2}$, for a FAR equal to 0.001 and for several values of the number of sensors. Note, for $N=1$, the much better performance of the OPT-LS receiver due to its capability to reject the rectilinear interference by phase discrimination between the sources. Note, for $2 \leq N \leq 4$, the better performance reached by the OPT-LS receiver, due to a better discrimination between the sources, done jointly by the phase and DOA, and despite of the fact that the CONV-LS receiver rejects the interference by a DOA discrimination. Thus, for rectilinear sources, software may replace sensors for given performances.

Note that when the interference considered previously corresponds to a useful path different from $i_{0}$, the detection performances of the useful path $i_{0}$ are still given by 
Figure 4(a) as long as the nonuseful path remains decorrelated from the useful path $i_{0}$. As the relative delay between the two paths decreases toward zero, the correlation between the two paths increases and the spatial filters $\widehat{\mathbf{w}}\left(l_{o} T_{e}\right)$ and $\widehat{\tilde{\mathbf{w}}}\left(l_{o} T_{e}\right)$ tend to keep the interferent path rather than to reject it. As a consequence, the power of the interference path tends to be added to that of the useful path for the detection process, hence a better detection of the useful path, as it is confirmed by simulations, nondescribed in the paper.

\section{EXTENSION TO MSK AND GMSK SIGNALS}

\subsection{Extension}

We briefly present in this section the extension of the previous results to MSK and GMSK modulations while a more detailed analysis of this problem will be presented elsewhere. The MSK and GMSK modulations [16] belong to the family of continuous phase modulation (CPM). It has been shown in [36] that GMSK modulation can be approximated by a linear modulation, while MSK is a linear modulation. In such conditions, the complex envelope of a useful MSK or GMSK signal takes the form

$$
s(t) \approx \sum_{n} j^{n} b_{n} f\left(t-n T-t_{s}\right)
$$

In (46), the approximated equality becomes a strict equality for a MSK signal, $b_{n}= \pm 1$ are the transmitted symbols if the latter are differentially encoded in the exact form of the modulation [19], $T$ is the symbol duration, $t_{s}\left(0 \leq t_{s} \leq T\right)$ is the time origin of the useful signal, and $f(t)$ is a real-valued pulse-shaped filter. This filter corresponds to

$$
f(t)=\left\{\begin{array}{cc}
\cos \left(\frac{\pi t}{2 T}\right) & -T \leq t \leq T \\
0 & \text { otherwise }
\end{array}\right\}
$$

for a MSK modulation whereas it may correspond either to the main pulse in Laurent's decomposition [36] or to the one computed in [19], which generates the best linear approximation of the GMSK in a least square sense. In both cases the temporal support of $f(t)$ for a GMSK modulation is about $4 T$ [19]. The derotation operation presented in [18, 19] consists to multiply the signal $s(t)$ by $j^{-t / T}$, giving rise to the derotated signal, $s_{d}(t)$, defined by

$$
s_{d}(t) \triangleq j^{-t / T} s(t) \approx \sum_{n} b_{n} f_{d}\left(t-n T-t_{s}\right),
$$

where $f_{d}(t) \triangleq j^{-\left(t+t_{s}\right) / T} f(t)$ is the equivalent pulse shaped filter of the derotated MSK or linearized GMSK signal. We deduce from (48) that $s_{d}(t)$ has the form of a BPSK signal but with two differences with respect to the latter. The first one is that $f_{d}(t)$ is no longer a $1 / 2$ Nyquist filter and intersymbol interference (ISI) will appear after a matched filtering operation to the filter $f_{d}(t)$. For this reason, the matched filtering operation to the pulse-shaped filter may not be required for the synchronization of MSK or GMSK signals. The second one is that $f_{d}(t)$ is no longer a real function but becomes a complex function. Thus, derotated MSK and GMSK signals may be interpreted as a BPSK signal which has been filtered by a nonideal complex propagation channel. For this reason, it has been shown in [17] that optimal WL spatial filters become sub-optimal for demodulation or synchronization of MSK or GMSK sources in the presence of interferences of the same form and that WL spatio-temporal (ST) filters are required. The number of taps per ST filter has to increase with the temporal support of $f(t) \otimes h(t)$, where $h(t)$ is the impulse response of the propagation channel.

ST WL filters with $L$ taps per filter are defined by

$$
\begin{aligned}
y_{\mathrm{WL}, \mathrm{ST}}\left(\left(\frac{l}{q}+n\right) T\right) \\
\triangleq \sum_{u=-(L-1) / 2}^{(L-1) / 2} \hat{\tilde{\mathbf{w}}}_{u}\left(l T_{e}\right)^{\dagger} \tilde{\mathbf{x}}_{d}\left(\left(\frac{l}{q}+n-u\right) T\right) \\
\triangleq \hat{\tilde{\mathbf{w}}}_{s t}\left(l T_{e}\right)^{\dagger} \tilde{\mathbf{x}}_{d, s t}\left(\left(\frac{l}{q}+n\right) T\right)
\end{aligned}
$$

if $L$ is odd and

$$
\begin{aligned}
y_{\mathrm{WL}, \mathrm{ST}} & \left(\left(\frac{l}{q}+n\right) T\right) \\
\triangleq & \sum_{u=-L / 2}^{L / 2-1} \hat{\tilde{\mathbf{w}}}_{u}\left(l T_{e}\right)^{\dagger} \tilde{\mathbf{x}}_{d}\left(\left(\frac{l}{q}+n-u\right) T\right) \\
\triangleq & \hat{\tilde{\mathbf{w}}}_{s t}\left(l T_{e}\right)^{\dagger} \tilde{\mathbf{x}}_{d, s t}\left(\left(\frac{l}{q}+n\right) T\right)
\end{aligned}
$$

if $L$ is even. In these expressions, $\hat{\widetilde{\mathbf{w}}}_{u}\left(l T_{e}\right)$ are $(2 N \times 1)$ spatial filters, $\mathbf{x}_{d}(t) \triangleq j^{-t / T} \mathbf{x}(t), \tilde{\mathbf{x}}_{d}\left(k T_{e}\right) \triangleq\left[\mathbf{x}_{d}\left(k T_{e}\right)^{\mathrm{T}}, \mathbf{x}_{d}\left(k T_{e}\right)^{\dagger}\right]^{\mathrm{T}}$, $\tilde{\mathbf{x}}_{d, s t}\left(k T_{e}\right)$ and $\hat{\tilde{\mathbf{w}}}_{s t}\left(l T_{e}\right)$ are $(2 L N \times 1)$ vectors defined by $\tilde{\mathbf{x}}_{d, s t}\left(k T_{e}\right) \triangleq\left[\tilde{\mathbf{x}}_{d}((k / q+(L-1) / 2) T)^{\mathrm{T}}, \ldots, \tilde{\mathbf{x}}_{d}((k / q-\right.$ $\left.(L-1) / 2) T)^{\mathrm{T}}\right]^{\mathrm{T}}$ and $\hat{\tilde{\mathbf{w}}}_{s t}\left(l T_{e}\right) \triangleq\left[\hat{\tilde{\mathbf{w}}}_{-(L-1) / 2}\left(l T_{e}\right)^{\mathrm{T}}, \ldots\right.$, $\left.\hat{\tilde{\mathbf{w}}}_{(L-1) / 2}\left(l T_{e}\right)^{\mathrm{T}}\right]^{\mathrm{T}}$, respectively, if $L$ is odd and by $\tilde{\mathbf{x}}_{d, s t}\left(k T_{e}\right) \triangleq$ $\left[\tilde{\mathbf{x}}_{d}((k / q+L / 2) T)^{\mathrm{T}}, \ldots, \tilde{\mathbf{x}}_{d}((k / q-L / 2) T)^{\mathrm{T}}\right]^{\mathrm{T}}$ and $\hat{\tilde{\mathbf{w}}}_{s t}\left(l T_{e}\right) \triangleq$ $\left[\hat{\tilde{\mathbf{w}}}_{-L / 2}\left(l T_{e}\right)^{\mathrm{T}}, \ldots, \hat{\tilde{\mathbf{w}}}_{L / 2}\left(l T_{e}\right)^{\mathrm{T}}\right]^{\mathrm{T}}$, respectively, if $L$ is even. The vector $\hat{\tilde{\mathbf{w}}}_{s t}\left(l T_{e}\right)$ minimizes the LS criterion (4) where $y_{v \mathrm{WL}}((l / q+n) T)=\hat{\tilde{\mathbf{w}}}\left(l T_{e}\right)^{\dagger} \tilde{\mathbf{x}}_{v}((l / q+n) T)$ is replaced by $y_{\mathrm{WL}, \mathrm{ST}}((l / q+n) T)$. In these conditions, it is straightforward to show that for MSK and GMSK signals, an LS approach using WL ST filters gives rise to (9) but with $\tilde{\mathbf{x}}_{d, s t}((l / q+n) T)$ instead of $\widetilde{\mathbf{x}}_{v}((l / q+n) T)$.

\subsection{Performance}

To compute and illustrate the performance of the OPT-LS receiver for MSK and GMSK signals, we consider the scenario of Figure 4(a) where BPSK sources have been replaced by either MSK (M) or GMSK (G) sources and we limit the analysis to the one sensor case $(N=1)$. For the OPT-LS receiver, we choose $L=1$ or $L=3$ taps whereas only 1 tap is chosen for the CONV-LS receiver. Under these assumptions, Figure 4(b) shows the probability of nondetection of the optimal delay $l_{o} T_{e}$ by the CONV-LS and OPT-LS receivers as a function of 
the input SNR, $\mu_{s}{ }^{2} \pi_{s} / \eta_{2}$, for a FAR equal to 0.001 . Note the poor performance of both CONV-LS receiver and OPT-LS receiver for $L=1$ and the good performance of OPT-LS receiver for $L=3$ for both modulations, showing off the capability of the OPT-LS receiver to do SAIC for both MSK and GMSK signals provided ST WL filters are used. Note finally the better performance of the OPT-LS receiver for MSK signals due to a smaller time support of the pulse-shaped filter. More insights about optimal values of $L$, partially given in [17] for channels with no delay spread, will be discussed elsewhere whatever the delay spread of the channel.

\section{CONCLUSION}

It has been shown in this paper that taking into account the noncircularity property of rectilinear interferences may dramatically improve the performance of both mono- and multichannels receivers for the synchronization of a BPSK signal in a radio communication network using this modulation. This result also holds for other rectilinear modulations such as AM or ASK modulations. For such signals and noncircular interferences, the optimal receiver, called OPT-LS receiver, has been shown to implement an optimal, in an LS sense, WL spatial filtering of the data followed by a correlation operation with a training sequence. Conditions, not required in practice, under which this optimal receiver becomes optimal for detection, in terms of GLRT approach, have also been given. A simplified performance analysis of both the conventional and the optimal receiver has been presented, allowing to prove in particular the ability of OPT-LS receiver to do single antenna interference cancellation and to show a decrease of the number of sensors for given performances. Besides, new analytical results about the convergence of the SINR at the output of both the SMF and the WL SMF, implemented from a training sequence, has been deduced from simulations. Extensions of the main results of the paper to both MSK and GMSK modulations have been briefly presented at the end of the paper. High performance of the OPT-LS receiver for these modulations have been obtained jointly with its capability to implement SAIC provided ST WL filters are used instead of spatial ones.

\section{APPENDIX}

It is shown in this appendix that expression (9) for $l=l_{o}$ is a sufficient statistic for the optimal detection, in the GLRT sense, of the known signal $s_{v}(n T)(0 \leq n \leq K-1)$ from the observation vectors $\mathbf{x}_{v}\left(\left(l_{o} / q+n\right) T\right)(0 \leq n \leq K-1)$, assuming that assumptions (A1) to (A7) are verified. To this aim, let us first compute the ML estimates of $\mu_{s} \widetilde{\mathbf{h}}_{s}$ and of $R_{\widetilde{b}}\left(l_{o} T_{e}\right)$ under $\mathrm{H} 1$ and $\mathrm{H} 0$, respectively. To do so, let us consider the likelihood of the parameters $s_{v}(n T)(0 \leq n \leq K-1), \mu_{s} \widetilde{\mathbf{h}}_{s}$, $R_{\tilde{b}}\left(l_{o} T_{e}\right)$ under H1, observing $\tilde{\mathbf{x}}_{v}\left(\left(l_{o} / q+n\right) T\right)(0 \leq n \leq K-1)$. Under the previous assumptions, this likelihood, $L_{1}\left(l_{o} T_{e}, K\right)$, can be written as

$$
L_{1}\left(l_{o} T_{e}, K\right) \triangleq \mathrm{p}\left[G^{\prime}\right]
$$

(where $G^{\prime}=\left\{\widetilde{\mathbf{x}}_{v}\left(\left(l_{o} / q+n\right) T\right)=\mu_{s} s_{v}(n T) \tilde{\mathbf{h}}_{s}+\tilde{\mathbf{b}}_{T v}\left(\left(l_{o} / q+n\right) T\right) /\right.$ $\left.\left.s_{v}(n T), \mu_{s} \tilde{\mathbf{h}}_{s}, R_{\widetilde{b}}\left(l_{o} T_{e}\right), 0 \leq n \leq K-1\right\}\right)$. Under the previous assumptions (A1) to (A7), expression (A.1) can be written as

$$
L_{1}\left(l_{o} T_{e}, K\right)=\prod_{n=0}^{K-1} \mathrm{p}\left[J_{n}^{\prime}\right]
$$

(where $J_{n}^{\prime}=\left\{\tilde{\mathbf{b}}_{T v}\left(\left(l_{o} / q+n\right) T\right)=\tilde{\mathbf{x}}_{v}\left(\left(l_{o} / q+n\right) T\right)-\mu_{s} s_{v}(n T) \tilde{\mathbf{h}}_{s} /\right.$ $\left.\left.s_{v}(n T), \mu_{s} \tilde{\mathbf{h}}_{s}, R_{\widetilde{b}}\left(l_{o} T_{e}\right)\right\}\right)$, and $\mathrm{p}\left[\tilde{\mathbf{b}}_{T v}\left(\left(l_{o} / q+n\right) T\right)\right]$ is defined by (15). Using (15) into (A.2) and taking the logarithm of $L_{1}\left(l_{o} T_{e}, K\right)$, we obtain

$$
\begin{aligned}
\log \left[L_{1}\right. & \left.\left(l_{o} T_{e}, K\right)\right] \\
= & -N K \log (\pi)-\left(\frac{K}{2}\right) \log \left(\operatorname{det}\left[R_{\tilde{b}}\left(l_{o} T_{e}\right)\right]\right) \\
& -\left(\frac{1}{2}\right) \sum_{n=0}^{K-1}\left[\tilde{\mathbf{x}}_{v}\left(\left(\frac{l_{o}}{q}+n\right) T\right)-\mu_{s} s_{v}(n T) \tilde{\mathbf{h}}_{s}\right]^{\dagger} \\
& \times R_{\widetilde{b}}\left(l_{o} T_{e}\right)^{-1}\left[\tilde{\mathbf{x}}_{v}\left(\left(\frac{l_{o}}{q}+n\right) T\right)-\mu_{s} s_{v}(n T) \tilde{\mathbf{h}}_{s}\right] .
\end{aligned}
$$

Using the fact that $\left|s_{v}(n T)\right|^{2}=r(0)^{2} \triangleq \pi_{s}$, it is then straightforward to show that the ML estimate, $\widehat{\mu}_{s} \hat{\tilde{\mathbf{h}}}_{s}$, of $\mu_{s} \widetilde{\mathbf{h}}_{s}$, that is, the estimate $\hat{\mu}_{s} \widehat{\widetilde{h}}_{s}$ which maximizes (A.3) is given by

$$
\hat{\mu}_{s} \hat{\tilde{\mathbf{h}}}_{s}=\left(\frac{1}{K \pi_{s}}\right) \sum_{n=0}^{K-1} \tilde{\mathbf{x}}_{v}\left(\left(\frac{l_{o}}{q}+n\right) T\right) s_{v}(n T)^{*} .
$$

Replacing $\mu_{s} \widetilde{\mathbf{h}}_{s}$ by $\hat{\mu}_{s} \hat{\widetilde{h}}_{s}$ into (A.3), it is well known $[8,10]$ that the ML estimate, $\hat{R}_{\widetilde{b} 1}\left(l_{o} T_{e}\right)$, of $R_{\widetilde{b}}\left(l_{o} T_{e}\right)$ under $\mathrm{H} 1$, that is, the matrix $\hat{R}_{\widetilde{b} 1}\left(l_{o} T_{e}\right)$ which maximizes (A.3) is given by

$$
\begin{aligned}
\hat{R}_{\tilde{b} 1}\left(l_{o} T_{e}\right)=\frac{1}{K} \sum_{n=0}^{K-1}[ & \left.\left.\tilde{\mathbf{x}}_{v}\left(\frac{l_{o}}{q}+n\right) T\right)-s_{v}(n T) \hat{\mu}_{s} \hat{\widetilde{\mathbf{h}}}_{s}\right] \\
\times & {\left[\tilde{\mathbf{x}}_{v}\left(\left(\frac{l_{o}}{q}+n\right) T\right)-s_{v}(n T) \hat{\mu}_{s} \hat{\tilde{\mathbf{h}}}_{s}\right]^{\dagger} . }
\end{aligned}
$$

In a similar way, it is straightforward to show that the ML estimate, $\hat{R}_{\tilde{b} 0}\left(l_{o} T_{e}\right)$, of $R_{\widetilde{b}}\left(l_{o} T_{e}\right)$ under $\mathrm{H} 0$ is given by

$$
\widehat{R}_{\tilde{b} 0}\left(l_{o} T_{e}\right)=\frac{1}{K} \sum_{n=0}^{K-1} \tilde{\mathbf{x}}_{v}\left(\left(\frac{l_{o}}{q}+n\right) T\right) \tilde{\mathbf{x}}_{v}\left(\left(\frac{l_{o}}{q}+n\right) T\right)^{\dagger} .
$$

On the other hand, using (A.5) into (A.3), we obtain, under $\mathrm{H} 1$,

$$
\begin{gathered}
\sum_{n=0}^{K-1}\left[\tilde{\mathbf{x}}_{v}\left(\left(\frac{l_{o}}{q}+n\right) T\right)-s_{v}(n T) \hat{\mu}_{s} \hat{\widetilde{h}}_{s}\right]^{\dagger} \widehat{R}_{\tilde{b} 1}\left(l_{o} T_{e}\right)^{-1} \\
\times\left[\tilde{\mathbf{x}}_{v}\left(\left(\frac{l_{o}}{q}+n\right) T\right)-s_{v}(n T) \hat{\mu}_{s} \hat{\widetilde{\mathbf{h}}}_{s}\right] \\
=K \operatorname{Tr}\left[\hat{R}_{\tilde{b} 1}\left(l_{o} T_{e}\right)^{-1} \hat{R}_{\tilde{b} 1}\left(l_{o} T_{e}\right)\right]=N K .
\end{gathered}
$$


In a similar way, we obtain, under $\mathrm{H} 0$,

$$
\begin{gathered}
\sum_{n=0}^{K-1} \mathbf{x}_{v}\left(\left(\frac{l_{o}}{q}+n\right) T\right)^{\dagger} \hat{R}_{\tilde{b} 0}\left(l_{o} T_{e}\right)^{-1} \mathbf{x}_{v}\left(\left(\frac{l_{o}}{q}+n\right) T\right) \\
=K \operatorname{Tr}\left[\widehat{R}_{\tilde{b} 0}\left(l_{o} T_{e}\right)^{-1} \widehat{R}_{\tilde{b} 0}\left(l_{o} T_{e}\right)\right]=N K .
\end{gathered}
$$

Then, using (15) into (14), replacing $R_{\widetilde{b}}\left(l_{o} T_{e}\right)$ by $\hat{R}_{\tilde{b} 0}\left(l_{o} T_{e}\right)$ under H0, $\mu_{s} \widetilde{\mathbf{h}}_{s}$ by $\hat{\mu}_{s} \hat{\widetilde{h}}_{s}$ and $R_{\widetilde{b}}\left(l_{o} T_{e}\right)$ by $\hat{R}_{\widetilde{b} 1}\left(l_{o} T_{e}\right)$ under H1 and using (A.7) and (A.8), it is straightforward to show that the likelihood receiver, $\operatorname{LR}\left(l_{o} T_{e}, K\right)$, defined by (12), takes the form

$$
\operatorname{LR}\left(l_{o} T_{e}, K\right)=\left(\frac{\operatorname{det}\left[\widehat{R}_{\tilde{b} 0}\left(l_{o} T_{e}\right)\right]}{\operatorname{det}\left[\hat{R}_{\tilde{b} 1}\left(l_{o} T_{e}\right)\right]}\right)^{K}
$$

where $\operatorname{det}(A)$ means determinant of matrix $A$. Moreover, we deduce from (A.4), (A.5), and (A.6) that

$$
\begin{aligned}
& \hat{R}_{\tilde{b} 1}\left(l_{o} T_{e}\right) \\
&=\hat{R}_{\tilde{b} 0}\left(l_{o} T_{e}\right)-\pi_{s}\left(\hat{\mu}_{s} \hat{\widetilde{h}}_{s}\right)\left(\hat{\mu}_{s} \hat{\widetilde{h}}_{s}\right)^{\dagger} \\
&=\hat{R}_{\tilde{b} 0}\left(l_{o} T_{e}\right)^{1 / 2}\left[I-\pi_{s} \hat{R}_{\tilde{b} 0}\left(l_{o} T_{e}\right)^{-1 / 2}\left(\hat{\mu}_{s} \hat{\widetilde{h}}_{s}\right)\right. \\
&\left.\quad \times\left(\hat{\mu}_{s} \hat{\widetilde{h}}_{s}\right)^{\dagger} \hat{R}_{\tilde{b} 0}\left(l_{o} T_{e}\right)^{-\dagger / 2}\right] \hat{R}_{\tilde{b} 0}\left(l_{o} T_{e}\right)^{\dagger / 2},
\end{aligned}
$$

where $\hat{R}_{\widetilde{b} 0}\left(l_{o} T_{e}\right)^{1 / 2}$ is a square root of $\hat{R}_{\widetilde{b}_{0}}\left(l_{o} T_{e}\right)$ such that $\hat{R}_{\tilde{b} 0}\left(l_{o} T_{e}\right)=\widehat{R}_{\widetilde{b} 0}\left(l_{o} T_{e}\right)^{1 / 2} \widehat{R}_{\widetilde{b} 0}\left(l_{o} T_{e}\right)^{\dagger / 2}, \widehat{R}_{\tilde{b} 0}\left(l_{o} T_{e}\right)^{\dagger / 2} \triangleq$ $\left(\widehat{R}_{\tilde{b}_{0}}\left(l_{o} T_{e}\right)^{1 / 2}\right)^{\dagger}, \widehat{R}_{\widetilde{b}_{0}}\left(l_{o} T_{e}\right)^{-\dagger / 2} \triangleq\left(\widehat{R}_{\tilde{b}_{0}}\left(l_{o} T_{e}\right)^{-1 / 2}\right)^{\dagger}$. Taking the determinant of the two sides of (A.10) and using the fact that $\operatorname{det}\left[I-\mathbf{u u}^{\dagger}\right]=1-\mathbf{u}^{\dagger} \mathbf{u}$, we obtain

$$
\begin{aligned}
& \operatorname{det} {\left[\widehat{R}_{\tilde{b} 1}\left(l_{o} T_{e}\right)\right] } \\
&=\operatorname{det}\left[\hat{R}_{\tilde{b} 0}\left(l_{o} T_{e}\right)\right]\left[1-\pi_{s}\left(\hat{\mu}_{s} \hat{\widetilde{h}}_{s}\right)^{\dagger} \hat{R}_{\tilde{b} 0}\left(l_{o} T_{e}\right)^{-1}\left(\hat{\mu}_{s} \hat{\widetilde{\mathbf{h}}}_{s}\right)\right]
\end{aligned}
$$

Using (A.11) into (A.9) we finally obtain

$$
\operatorname{LR}\left(\mathbf{x}_{v}\right)\left(l_{o} T_{e}, K\right)=\left(\frac{1}{\left[1-\pi_{s}\left(\hat{\mu}_{s} \hat{\widetilde{h}}_{s}\right)^{\dagger} \hat{R}_{\tilde{b}_{0}}\left(l_{o} T_{e}\right)^{-1}\left(\hat{\mu}_{s} \hat{\tilde{\mathbf{h}}}_{s}\right)\right]}\right)^{K}
$$

which shows that a sufficient statistic for the optimal detection of the known signal $s_{v}(n T)(0 \leq n \leq K-1)$ from the observations $\mathbf{x}_{v}\left(\left(l_{o} / q+n\right) T\right)(0 \leq n \leq K-1)$, assuming (A1) to (A7), is given by

$$
\begin{aligned}
\hat{C}_{\mathrm{OPT}-\mathrm{LS}}\left(l_{o} T_{e}, K\right) & =\pi_{s}\left(\hat{\mu}_{s} \hat{\tilde{\mathbf{h}}}_{s}\right)^{\dagger} \hat{R}_{\widetilde{b}_{0}}\left(l_{o} T_{e}\right)^{-1}\left(\hat{\mu}_{s} \hat{\tilde{\mathbf{h}}}_{s}\right) \\
& =\left(\frac{1}{\pi_{s}}\right) \hat{\mathbf{r}}_{\tilde{x} s}\left(l_{o} T_{e}\right)^{\dagger} \hat{R}_{\tilde{x}}\left(l_{o} T_{e}\right)^{-1} \hat{\mathbf{r}}_{\tilde{x} s}\left(l_{o} T_{e}\right) .
\end{aligned}
$$

\section{REFERENCES}

[1] S. E. Bensley and B. Aazhang, "Subspace-based channel estimation for code division multiple access communication systems," IEEE Transactions on Communications, vol. 44, no. 8, pp. 1009-1020, 1996.

[2] S. E. Bensley and B. Aazhang, "Maximum-likelihood synchronization of a single user for code-division multiple-access communication systems," IEEE Transactions on Communications, vol. 46, no. 3, pp. 392-399, 1998.

[3] U. Madhow, "Blind adaptive interference suppression for the near-far resistant acquisition and demodulation of directsequence CDMA signals," IEEE Transactions on Signal Processing, vol. 45, no. 1, pp. 124-136, 1997.

[4] U. Madhow, "Blind adaptive interference suppression for direct-sequence CDMA," Proceedings of the IEEE, vol. 86, no. 10, pp. 2049-2069, 1998.

[5] A. Mantravadi and V. V. Veeravalli, "Multiple-access interference-resistant acquisition for band-limited CDMA systems with random sequences," IEEE Journal on Selected Areas in Communications, vol. 18, no. 7, pp. 1203-1213, 2000.

[6] E. G. Ström, S. Parkvall, S. L. Miller, and B. E. Ottersten, "Propagation delay estimation in asynchronous directsequence code-division multiple access systems," IEEE Transactions on Communications, vol. 44, no. 1, pp. 84-93, 1996.

[7] D. Zheng, J. Li, S. L. Miller, and E. G. Ström, "An efficient codetiming estimator for DS-CDMA signals," IEEE Transactions on Signal Processing, vol. 45, no. 1, pp. 82-89, 1997.

[8] L. E. Brennan and I. S. Reed, "An adaptive array signal processing algorithm for communications," IEEE Transactions on Aerospace and Electronic Systems, vol. 18, no. 1, pp. 124-130, 1982.

[9] R. T. Compton Jr., "An adaptive array in a spread-spectrum communication system," Proceedings of the IEEE, vol. 66, no. 3, pp. 289-298, 1978.

[10] D. M. Dlugos and R. A. Scholtz, "Acquisition of spread spectrum signals by an adaptive array," IEEE Transactions on Acoustics, Speech, and Signal Processing, vol. 37, no. 8, pp. 12531270, 1989.

[11] B. Wang and H. M. Kwon, "PN code acquisition using smart antenna for spread-spectrum wireless communications-part I," IEEE Transactions on Vehicular Technology, vol. 52, no. 1, pp. 142-149, 2003.

[12] J. H. Winters, "Spread spectrum in a four-phase communication system employing adaptive antennas," IEEE Transactions on Communications, vol. 30, no. 5, part 2, pp. 929-936, 1982.

[13] B. Picinbono, "On circularity," IEEE Transactions on Signal Processing, vol. 42, no. 12, pp. 3473-3482, 1994.

[14] F. D. Neeser and J. L. Massey, "Proper complex random processes with applications to information theory," IEEE Transactions on Information Theory, vol. 39, no. 4, pp. 1293-1302, 1993.

[15] S. Sfar and K. B. Letaief, "Improved group multiuser detection with multiple receive antennas in the presence of improper multi-access interference," IEEE Transactions on Communications, vol. 53, no. 4, pp. 560-563, 2005.

[16] J. G. Proakis, Digital Communications, McGraw-Hill, New York, NY, USA, 3rd edition, 1995.

[17] P. Chevalier and F. Pipon, "New insights into optimal widely linear array receivers for the demodulation of BPSK, MSK, and GMSK signals corrupted by noncircular 
interferences-application to SAIC," IEEE Transactions on Signal Processing, vol. 54, no. 3, pp. 870-883, 2006.

[18] Z. Ding and G. Li, "Single-channel blind equalization for GSM cellular systems," IEEE Journal on Selected Areas in Communications, vol. 16, no. 8, pp. 1493-1505, 1998.

[19] H. Trigui and D. T. M. Slock, "Performance bounds for cochannel interference cancellation within the current GSM standard," Signal Processing, vol. 80, no. 7, pp. 1335-1346, 2000.

[20] P. Chevalier, F. Pipon, and F. Delaveau, "Procédé et dispositif de synchronisation de liaisons rectilignes ou quasirectilignes en présence d'interférences de même nature," Patent FR.05.01784, February 2005.

[21] B. Picinbono and P. Chevalier, "Widely linear estimation with complex data," IEEE Transactions on Signal Processing, vol. 43, no. 8, pp. 2030-2033, 1995.

[22] W. Brown and R. Crane, "Conjugate linear filtering," IEEE Transactions on Information Theory, vol. 15, no. 4, pp. 462465, 1969.

[23] W. A. Gardner, "Cyclic Wiener filtering: theory and method," IEEE Transactions on Communications, vol. 41, no. 1, pp. 151163, 1993.

[24] W. A. Gardner, Cyclostationarity in Communications and Signal Processing, IEEE Press, New York, NY, USA, 1994.

[25] P. Chevalier, "Filtrage d'antenne optimal pour signaux non stationnaires-concepts, performances," in Proceedings of the 15th GRETSI Symposium on Signal and Image Processing, pp. 233-236, Juan-Les-Pins, France, September 1995.

[26] P. Chevalier, "Optimal array processing for non stationary signals," in Proceedings of IEEE International Conference on Acoustics, Speech, and Signal Processing (ICASSP '96), vol. 5, pp. 2868-2871, Atlanta, Ga, USA, May 1996.

[27] P. Ciblat, P. Loubaton, E. Serpedin, and G. B. Giannakis, "Performance analysis of blind carrier frequency offset estimators for noncircular transmissions through frequency-selective channels," IEEE Transactions on Signal Processing, vol. 50, no. 1, pp. 130-140, 2002.

[28] P. Ciblat, E. Serpedin, and Y. Wang, "On a blind fractionally sampling-based carrier frequency offset estimator for noncircular transmissions," IEEE Signal Processing Letters, vol. 10, no. 4, pp. 89-92, 2003.

[29] A. Napolitano and M. Tanda, "Doppler-channel blind identification for noncircular transmissions in multiple-access systems," IEEE Transactions on Communications, vol. 52, no. 12, pp. 2073-2078, 2004.

[30] P. Chevalier and F. Pipon, "Optimal array receiver for synchronization of a BPSK signal corrupted by non circular interferences," in Proceedings of IEEE International Conference on Acoustics, Speech, and Signal Processing (ICASSP '06), vol. 4, pp. 1061-1064, Toulouse, France, May 2006.

[31] H. L. van Trees, Detection, Estimation and Modulation Theory_Part I, John Wiley \& Sons, New York, NY, USA, 1968.

[32] F. Delaveau, F. Pipon, and O. Lambron, "Smart antennas for interference resolution in cellular networks," in Proceedings of the 14th International Wroclaw Symposium and Exhibition on Electromagnetic Compatibility, pp. 264-267, Wroclaw, Poland, June 1998.

[33] B. Picinbono, "Second-order complex random vectors and normal distributions," IEEE Transactions on Signal Processing, vol. 44, no. 10, pp. 2637-2640, 1996.
[34] A. van den Bos, "The multivariate complex normal distribution-a generalization," IEEE Transactions on Information Theory, vol. 41, no. 2, pp. 537-539, 1995.

[35] R. A. Monzingo and T. W. Miller, Introduction to Adaptive Arrays, John Wiley \& Sons, New York, NY, USA, 1980.

[36] P. A. Laurent, "Exact and approximate construction of digital phase modulations by superposition of amplitude modulated pulses (AMP)," IEEE Transactions on Communications, vol. 34, no. 2, pp. 150-160, 1986.

Pascal Chevalier received the M.S. degree from Ecole Nationale Supérieure des Techniques Avancées (ENSTA) and the Ph.D. degree from South-Paris University, France, in 1985 and 1991, respectively. Since 1991 he has been with Thomson-CSF/RGS (now Thalés-Communications) where has shared industrial activities (studies, experimentations, expertises, management), teaching activities both in French engineer schools (ESE, ENST, ENSTA) and French universities (Cergy-Pontoise) and research activities. Since 2000, he has also been acting as a Technical Manager and Architect of the array processing sub-system as part of a national program of military satellite telecommunications. He is currently a Thalés Expert since 2003. His present research interests are in array processing techniques for applications such as radiocommunications networks, satellite telecommunications, spectrum monitoring, and passive listening in HVUHF band. He has been a Member of the THOMSON-CSF Technical and Scientifical Council between 1995 and 1998. He coreceived the 2003 "Science and Defense" Award from the French Ministry of Defence for its work as a whole about array processing for military radiocommunications. He is author or coauthor of about 20 patents and 100 papers. He is presently an EURASIP member and an emeritus Member of the Societé des Electriciens et des Electroniciens (SEE).

François Pipon was born in 1964 in Melle (Deux-Sèvres), France. He received the M.S. degree both from Ecole Polytechnique and Ecole Nationale Supérieure des Techniques Avancées (ENSTA) in 1987 and 1989, respectively. In 1989, he joined Thomson CSF/RGS (now Thalès-Communications) as an Engineer in the field of array processing, working on both direction finding and adaptive array filtering aspects. Since

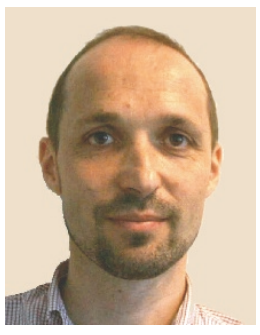
1993, he has shared industrial activities (studies, experimentations, expertises, management, etc.), and research activities. Since 1998, he has worked on a multichannel interference analyser product (SMART AIR) for the GSM network. He has developed most of the antenna processing techniques implemented in the equipment. His present research interests are in multichannel equalizer techniques, for applications such as TDMA (especially the GSM network) and CDMA radiocommunications networks, satellite telecommunications, HF telecommunications, spectrum monitoring, and HF/VUHF passive listening. He coreceived the 2003 "Science and Defense" Award from the French Ministry of Defence for its work as a whole about array processing for military radiocommunications. He is author or coauthor of 14 patents and more than 20 papers (journal and conferences). 
François Delaveau was born in 1964 in Argenteuil, France, he received the M.S. degrees from Ecole Nationale Supérieure de Techniques Avancées (ENSTA), Paris, France, in 1987, and from Mathematics University (Maîtrise and Agrégation - 1988 and 1990). Since 1987, he shares industrial activities (studies, experimentation, management, etc.) and research activities. After working in RADAR, SONAR, infra-red, and

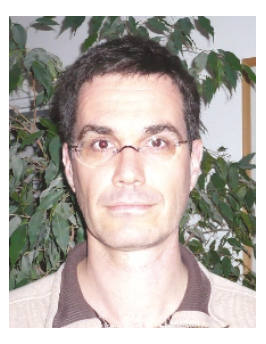
acoustic systems for various advanced militarian applications, he joined Thomson CSF/COMSYS in 1997 to develop a new line of RF instruments for spectrum monitoring applications. He was involved either in signal processing applications focused on terrestrial and satellite transmissions, in adaptive array techniques dedicated to interference measurement within OFDM, TDMA, and CDMA networks, in advanced developments related to COMINT applications, regarding especially radiocellulars and civilian standards. He is currently a Thales expert for radiocellulars and he manages a laboratory of THALES communication that is dedicated to signal analysis and antenna processing. He is author or coauthor of many papers, ITU recommendations, and Thalés patents for signal measurement. 